\title{
Lilly's Model for Steady-State Tropical Cyclone Intensity and Structure $\mathscr{O}$
}

\author{
DANDAN TAO, ${ }^{\mathrm{a}}$ RiChaRd RotunnO, ${ }^{\mathrm{b}}$ AND MiCHAEl Bell ${ }^{\mathrm{a}}$ \\ ${ }^{a}$ Department of Atmospheric Science, Colorado State University, Fort Collins, Colorado \\ ${ }^{\mathrm{b}}$ National Center for Atmospheric Research, Boulder, Colorado
}

(Manuscript received 27 February 2020, in final form 20 August 2020)

\begin{abstract}
This study revisits the axisymmetric tropical cyclone (TC) theory from D. K. Lilly's unpublished manuscript (Lilly model) and compares it to axisymmetric TC simulations from a nonhydrostatic cloud model. Analytic solutions of the Lilly model are presented through simplifying assumptions. Sensitivity experiments varying the sea surface, boundary layer and tropopause temperatures, and the absolute angular momentum $(M)$ at some outer radius in the Lilly model show that these variations influence the radial structure of the tangential wind profile $V(r)$ at the boundary layer top. However, these parameter variations have little effect on the inner-core normalized tangential wind, $V\left(r / r_{m}\right) / V_{m}$, where $V_{m}$ is the maximum tangential wind at radius $r_{m}$. The outflow temperature $T_{\infty}$ as a function of $M$ (or saturation entropy $s^{*}$ ) is found to be the only input that changes the normalized tangential wind radial structure in the Lilly model. In contrast with the original assumption of the Lilly model that $T_{\infty}\left(s^{*}\right)$ is determined by the environment, it is argued here that $T_{\infty}\left(s^{*}\right)$ is determined by the TC interior flow under the environmental constraint of the tropopause height. The present study shows that the inner-core tangential wind radial structure from the Lilly model generally agrees well with nonhydrostatic cloud model simulations except in the eyewall region where the Lilly model tends to underestimate the tangential winds due to its balanced-dynamics assumptions. The wind structure in temperature-radius coordinates from the Lilly model can largely reproduce the numerical simulation results. Though the Lilly model is based on a number of simplifying assumptions, this paper shows its utility in understanding steady-state TC intensity and structure.
\end{abstract}

KEYWORDS: Dynamics; Tropical cyclones

\section{Introduction}

Tropical cyclones (TCs) are a severe hazard for coastal areas given their devastating power. Studies have shown that the amount of economic loss is not only because of TC intensity but also because of the associated rainfall amounts and storm surge, which depend on TC size and strength (Iman et al. 2005; Irish et al. 2008). Therefore, increasing attention is being directed to TC structure (Ying and Zhang 2012; Chan and Chan 2014; Xu and Wang 2010, 2015, 2018). Most of these studies use either numerical model simulations or statistical analysis of observational data. Though the theoretical work on maximum TC intensity has been quite well studied, there have been few attempts to develop a theory for TC structure (Shutts 1981; Emanuel 1986, hereafter E86; Emanuel 2004, hereafter E04; Emanuel and Rotunno 2011, hereafter ER11; Chavas et al. 2015, hereafter CLE15) or evaluate a TC structure theory from observational and modeling data (Stern and Nolan 2009; Tao et al. 2019). Numerical models today can quite realistically represent the 3D structure of TCs through all stages; however, the physical and mathematical understandings underlying the numerical-model-derived TC structures and evolutions are still lacking.

Given the circular structure of mature TCs, the axisymmetric assumption is a reasonably well justified simplification

Supplemental information related to this paper is available at the Journals Online website: https://doi.org/10.1175/JAS-D-200057.s1.

Corresponding author: Dandan Tao, ddantao@colostate.edu of the structure during TC development after slantwise neutrality is established (Peng et al. 2019), especially during the TC's rapid intensification and steady-state stages. The steadystate TC inner-core structure was theoretically considered in D. K. Lilly (1986, unpublished manuscript, see online supplement, hereafter L86) as well as in a series of other papers (E86; E04; ER11; CLE15). A detailed comparison of these theories is presented in section 2. One outstanding contribution of L86 is the explicit introduction of the outflow temperature $T_{\infty}$ as a function of entropy $s$ (or absolute angular momentum $M$ ) to close the equations. In ER11, the authors hypothesize that $T_{\infty}(M)$ is determined by the TC vortex itself through smallscale turbulent mixing in the outflow that depends on a critical Richardson number. In contrast, L86 takes $T_{\infty}(s)$ as given by the environmental thermodynamic sounding. In either approach, the radial profile of tangential wind at the boundary layer top $V(r)$ is directly connected to the outflow temperature stratification $T_{\infty}(s)$. We will discuss the question of how $T_{\infty}(s)$ is determined in section 2 .

The purpose of this paper is to bring the unpublished L86 to light and further extend that work, which we believe will help in the understanding of steady-state TC structures. In this paper, we will present the derivations in L86 and examine the extent to which the theory can explain TC structure from numerical model simulations. Hereafter, we denote the theory developed in L86 as the Lilly model. This paper is organized as follows. Section 2 revisits the basic equations of the Lilly model and compares it with the existing theories presented in E86, E04, ER11, and CLE15. Section 3 introduces the analytic solutions for the governing equations in the Lilly model with further simplifications. Section 4 shows the dependence of the 
Lilly model solutions on different dynamic and thermodynamic input parameters. Section 5 compares the results from fullphysics model simulations with those from the Lilly model. Section 6 summarizes the present findings and discusses the potential application of the Lilly model in understanding TCs.

\section{The Lilly model}

\section{a. The Lilly model reconsidered}

Although the basic features of the Lilly model are available in the existing literature (Emanuel et al. 1985; Lilly and Emanuel 1985; E04), there are several aspects that are not available in these papers as they appeared in an earlier document (D. K. Lilly 1979, unpublished manuscript, hereafter L79; see supplement). We believe these aspects are useful for interpretation of the Lilly model and therefore we incorporate L79 into the following.

The principle assumptions of steady-state and frictionless flow above the TC boundary layer as well as axisymmetry and pseudoadiabatic thermodynamics (Bryan 2008) lead to the following equation:

$$
\frac{\eta}{\rho_{d} r}+\frac{1}{2 r^{2}} \frac{d M^{2}}{d \psi}+T \frac{d s}{d \psi}=\frac{d E}{d \psi}+\frac{d}{d \psi}\left(\frac{1}{2} f M\right),
$$

where $\rho_{d}$ is the density of dry air, $T$ is the absolute temperature, $f$ is the Coriolis parameter, $s$ is the actual entropy (for both unsaturated and saturated air), $\psi$ is the streamfunction defined by $\rho_{d} u=(1 / r) \partial \psi / \partial z$ and $\rho_{d} w=-(1 / r) \partial \psi / \partial r, M \equiv r v+(1 / 2) f r^{2}$, $\eta \equiv \partial u / \partial z-\partial w / \partial r, E \equiv(1 / 2)\left(u^{2}+v^{2}+w^{2}\right)+g z+c_{p} T+L_{o} q$. The expression for $E$ contains the wind components $(u, v, w)$ in the cylindrical coordinates $(r, \phi, z)$, gravity $(g)$, the water vapor mixing ratio $(q)$, the latent heat of vaporization $\left(L_{o}\right)$ and the specific heat of air at constant pressure $\left(c_{p}\right)$. A detailed derivation of (1) can be found in appendix B of Bryan and Rotunno (2009a).

The assumption of steady, inviscid, axisymmetric flow allows the $\phi$-momentum equation to be written as $J(\psi, M) \equiv$ $(\partial \psi / \partial r)(\partial M / \partial z)-(\partial \psi / \partial z)(\partial M / \partial r)=0$, which implies $M=M(\psi)$. Similarly, for a pseudoadiabatic moisture process we have $J(\psi, s)=0$ and therefore, $s=s(\psi)$. Also, for steady, inviscid, pseudoadiabatic, axisymmetric flow, it can be shown that $J(\psi, E)=0$, and therefore, $E=E(\psi)$. L79 observes that (1) is a "Long's equation" (Yih 1965, chapter 3), which is a nonlinear partial differential equation for $\psi$ since $\eta=\partial u / \partial z-\partial w / \partial r=$ $(\partial / \partial z)\left[\left(1 / \rho_{d} r\right)(\partial \psi / \partial z)\right]+(\partial / \partial r)\left[\left(1 / \rho_{d} r\right)(\partial \psi / \partial r)\right]$ with $M, s$ and $E$ all known functions of $\psi$ (the boundary conditions are therefore on $\psi)$. In the limit of constant entropy $(d s / d \psi=0)$, (1) has been used to describe rotating axial flow in tubes with $M(\psi)$ and $E(\psi)$ given upstream [e.g., Yih (1965), chapter 6.7; Batchelor (1967), chapter 7.5], while in the case of nonrotating $(M=0)$ density-stratified flow with $s(\psi)$ and $E(\psi)$ given upstream, (1) has been used to describe mountain waves (Yih 1965, chapter 3.7; Long 1955). In these examples, the functions $M, s$, and $E$ are considered known functions of $\psi$ at some upstream location.

In L79 and L86, the implications of the idealized TC obeying a type of Long's equation were not explored, but as shown here, they offer insights into questions surrounding the determination of $T_{\infty}(s)$. In the case of a tropical cyclone, the flow rising out of the boundary layer must per force be considered as "upstream." How can $M(\psi), s(\psi)$, and $E(\psi)$ be determined at the top of the boundary layer? The authors are not aware of any general method to determine these functions other than a boundary layer model or direct observation; however, the full form (1) allows the following inferences. In the limit as $r \rightarrow \infty$ (with finite numerators), the first two terms on the left-hand side of (1) vanish and (1) reduces to

$$
T_{\infty} \frac{d s}{d \psi}=\frac{d}{d \psi}\left(E+\frac{1}{2} f M\right),
$$

which implies $T_{\infty}=T_{\infty}(\psi)$. Since $M, s$, and $E$ are all functions of $\psi, T_{\infty}$ can be considered a function of any one of them; L86 chooses $T_{\infty}=T_{\infty}(s)$. Equation (2) makes it plain that taking (1) to the limit $r \rightarrow \infty$ does not eliminate the unknown function $(d / d \psi)[E+(1 / 2) f M]$ but merely transforms it to another unknown function, $T_{\infty}(s)$. L79 and L86 assumed that $T_{\infty}(s)$ can be taken from an unperturbed environmental sounding. However, (2) shows that the Lilly model requires an internal consistency between $T_{\infty}(s)$ and the distributions of $s$ and $E+(1 / 2) f M$ "upstream," or rather, at the top of the boundary layer at locations where the flow is upward. We return below to the discussion of $T_{\infty}(s)$.

Substituting (2) into (1) gives

$$
\frac{\eta}{\rho_{d} r}+\frac{1}{2 r^{2}} \frac{d M^{2}}{d \psi}=-\left[T-T_{\infty}(s)\right] \frac{d s}{d \psi},
$$

which, based on the foregoing discussion, we understand as applying to the interior flow that issues up from the boundary layer and subsequently flows radially outward while retaining the relations $M(\psi), s(\psi)$, and $E(\psi)$. L79 proceeds to neglect the first term on the left-hand side of (3) based on the hydrostatic and gradient wind approximations that reduces (3) to an ordinary differential equation; from a physical standpoint, the connection to the flow in the $r-z$ plane is no longer in evidence since the differential $d \psi$ drops out. Then we have

$$
M=-\left[T-T_{\infty}(s)\right] r^{2} \frac{d s}{d M},
$$

which simplifies (3), but does not change the relation (2), which says $T_{\infty}(s)$ must be compatible with upstream conditions on $M(\psi), s(\psi)$, and $E(\psi)$. In fact one can say, based on (2), that specifying the "downstream" $T_{\infty}(s)$ is tantamount to specifying the "upstream" $(d / d s)[E+(1 / 2) f M]$. The above discussion implies that the Lilly model only applies to the flow being upward from the boundary layer, which is mostly near the inner radii of the TC vortex. Since the Lilly model is only valid where the air flows out of the boundary layer, the air is mostly saturated and therefore we will use $s^{*}$ for the entropy in the following derivations.

Using a "slab" boundary layer model, one finds

$$
\frac{d s^{*}}{d M}=\frac{C_{k}}{C_{d}} \frac{s^{*}-s_{s}^{*}}{\left(M-\frac{1}{2} f r^{2}\right)}
$$


where $s_{s}^{*}$ is the saturation entropy of air in contact with the sea surface, $s^{*}$ is the saturation entropy at the boundary layer top, and $C_{k}$ and $C_{d}$ are the exchange coefficients for enthalpy and momentum, respectively.

Using (5) in (4) to solve for $r^{2}$, and focusing on the boundary layer top with assumed-constant temperature $T_{b}$, we obtain

$$
r^{2}=\frac{M}{\frac{1}{2} f+\frac{C_{k}}{C_{d}} \frac{\left(T_{b}-T_{\infty}\right)\left(s_{s}^{*}-s^{*}\right)}{M}} .
$$

Using (6) to replace $r^{2}$ in (5) yields

$$
\frac{d M}{d s^{*}}=-\frac{\left(T_{b}-T_{\infty}\right)}{\frac{1}{2} f+\frac{C_{k}}{C_{d}} \frac{\left(T_{b}-T_{\infty}\right)\left(s_{s}^{*}-s^{*}\right)}{M}}
$$

To obtain $s_{s}^{*}(r)$, the derivation proceeds from the first law of thermodynamics,

$$
T d s^{*}=c_{p} d T-\frac{d p}{\rho}+L_{o} d q^{*},
$$

where $q^{*}$ is the saturation mixing ratio and $p$ is the pressure. By approximating the saturation mixing ratio as $q^{*}=0.622 e(T) / p$, the differential can be represented as

$$
d q^{*}=0.622\left(\frac{d e}{p}-e \frac{d p}{p^{2}}\right)=q^{*}\left(\frac{d e}{e}-\frac{d p}{p}\right),
$$

where $e(T)$ is the saturation vapor pressure. For the idealized condition of a constant sea surface temperature, $d e=0$, since $d T_{s}=0$. Using (9) in (8) with a constant sea surface temperature yields

$$
T_{s} d s_{s}^{*}=-\frac{d p_{s}}{\rho_{s}}-L_{o} q_{s}^{*} \frac{d p_{s}}{p_{s}},
$$

where the subscript $s$ indicates the sea surface. Utilizing the ideal gas law, $p_{s}=\rho_{s} R T_{s}$, where $R$ is the gas constant for dry air, (10) becomes

$$
d s_{s}^{*}=-\gamma \times \frac{d p_{s}}{\rho_{s}},
$$

with

$$
\gamma \equiv\left(R T_{s}+L_{o} q_{s}^{*}\right) /\left(R T_{s}^{2}\right) .
$$

Last, gradient wind balance is used to link $s_{s}^{*}$ with $M$. In terms of the tangential velocity $V$, gradient wind balance is expressed as

$$
\frac{1}{\rho} \frac{\partial p}{\partial r}-\left(f+\frac{V}{r}\right) V=0
$$

which can be written in terms of $M$ as

$$
\frac{1}{\rho} \frac{\partial p}{\partial r}=\frac{M^{2}-f^{2} r^{4} / 4}{r^{3}}
$$

Assuming the pressure gradient at the boundary layer top is the same as the pressure gradient at the sea surface, we obtain $d s_{s}^{*} / d r$ by dividing (11) by $d r$ and then using (14) such that

$$
\frac{d s_{s}^{*}}{d r}=-\gamma \times\left(\frac{M^{2}-f^{2} r^{4} / 4}{r^{3}}\right) .
$$

With the further specification of

$$
T_{\infty}=T_{\infty}\left(s^{*}\right)
$$

\{or equivalently $\left.\left(d / d s^{*}\right)[E+(1 / 2) f M]\right\}$, the system of equations is closed since, in effect, the "upstream" (the top of the boundary layer) relations among $M, s^{*}$, and $E$ are specified by virtue of (2).

With $s^{*}$ as the independent variable, the four equations of (6), (7), (15), and (16) form a complete set in the dependent variables of $M, s_{s}^{*}, r$, and $T_{\infty}$. Given the inputs of $T_{b}$ and $M, s_{s}^{*}, s^{*}$ at some radius $r_{0}$ [determined by (6)] $\left(M_{0}, s_{s 0}^{*}, s_{0}^{*}\right), M, s_{s}^{*}, r$, and $T_{\infty}$ can be solved for numerically. These four equations constitute the Lilly model, which provides a theoretical solution for steadystate axisymmetric TC inner-core structure.

\section{b. The Lilly model compared with other similar models}

In a parallel development, (4) was derived by E86 on different grounds by assuming from the outset hydrostatic and gradient wind balance, and slantwise moist neutrality, which implies the saturation entropy $s^{*}=s^{*}(M)$. Both E86 and the Lilly model connect the interior equation, (4), to the boundary layer using the slab model, (5), which technically gives a relation between the unsaturated boundary layer $s$ and $M$. Section 3 of E04 reasons that $s$ inside the boundary layer equals $s^{*}$ at the boundary layer top only for upward motions from the boundary layer. Hence, although E86 and the Lilly model are derived on different grounds, both are only valid for flow upward from the boundary layer, which is mainly near the inner radii of the TC (Fig. 3 of E04).

Both approaches also share the uncertainty of how to specify $T_{\infty}\left(s^{*}\right)$. L86 assumed that $T_{\infty}\left(s^{*}\right)$ is set by the unperturbed environment. E86 distinguished an inner region where (4) and (5) are valid and assumed $T_{\infty}\left(s^{*}\right)=T_{o}$, a constant; E86 considered the outer region as characterized by radiativeconvective equilibrium with boundary layer relative humidity assumed to have a constant value of $80 \%$ (Fig. 5 of E86). ER11 attempted to improve the inner-region calculation of E86 by hypothesizing that $T_{\infty}\left(s^{*}\right)$ is internally determined through small-scale turbulent mixing in the outflow region and showed that the tangential velocity distribution at the top of the boundary layer $V(r)$ has a fundamental relation to $T_{\infty}\left(s^{*}\right)$ [ER11 used $T_{\infty}(M)$ ]. Their closure of $T_{\infty}(M)$ and small-scale mixing involves a number of simplifying assumptions of varying validity (Tao et al. 2019). One purpose of the present paper is to revisit some of the simple solutions given in L79 for $V(r)$ with simple assumed forms of $T_{\infty}\left(s^{*}\right)$. These simple solutions show that the fundamental connection between $T_{\infty}\left(s^{*}\right)$ and $V(r)$ at steady state does not depend on any particular physical model (such as small-scale instability) for $T_{\infty}\left(s^{*}\right)$, but rather, is a consequence of the assumptions underlying the basic model (4) and (5). Another purpose of this paper is to point out that the Lilly model, understood as a type of Long's equation, implies that $T_{\infty}\left(s^{*}\right)$ is determined from 
the TC interior, and in this aspect, is similar to ER11. We provide evidence for the validity of (2) in section $5 \mathrm{~d}$.

From the above discussion it is clear that the Lilly model and ER11 do not apply to the TC outer region where the motion is mostly downward into the boundary layer. The solution for the TC outer subsidence region has been presented in E04. CLE15 constructed a model for $V(r)$ by matching the E04 solution for the outer radii with the ER11 solution for the inner radii and found reasonable agreement with observations, especially in the outer region. A comparison of the Lilly model and the other models discussed here is summarized in Table 1. Note that the Lilly model presented in this study (TRB20) is built upon L79/L86 but has a different interpretation of $T_{\infty}\left(s^{*}\right)$.

\section{Analytic solutions of the Lilly model with further simplifications}

According to the discussion in the previous section, the outflow temperature $T_{\infty}\left(s^{*}\right)$ is determined by the upstream relation among $s^{*}, M$, and $E$, which is unknown. To demonstrate the connection between $T_{\infty}\left(s^{*}\right)$ and the upstream structure at the boundary layer top, we take $T_{\infty}\left(s^{*}\right)$ as a given function. In this section, analytic solutions of the Lilly model with further simplifications are provided. The inputs of $T_{b}, s_{s 0}^{*}, s_{0}^{*}$, and $M_{0}$ are $T_{b}=$ $269.87 \mathrm{~K}^{1}{ }^{1} s^{*}, s_{0}^{*}=5856.26 \mathrm{~J} \mathrm{~K}^{-1} \mathrm{~kg}^{-1}, s_{s 0}^{*}=5940.13 \mathrm{~J} \mathrm{~K}^{-1} \mathrm{~kg}^{-1}$, and $M_{0}=1.5 \times 10^{6} \mathrm{~m}^{2} \mathrm{~s}^{-1}$, which are chosen from a model simulation described in section 5. In practice, these inputs can be a set of any reasonable values drawn from climatology or any case study. The nonzero Coriolis parameter used in this section is $f=5 \times 10^{-5} \mathrm{~s}^{-1}$, while the nonzero $\gamma$ is $0.0056 \mathrm{~K}^{-1}$ calculated based on a sea surface temperature of $301.15 \mathrm{~K}$.

a. Linear $T_{\infty}\left(s^{*}\right)$ with $f=0$ and $\gamma=0$

A simplified version of the foregoing model can be obtained with $f=0$ and $\gamma=0$. The first assumption is well justified for the inner core of mature TCs, where $V \gg f r$ given $f=5 \times$ $10^{-5} \mathrm{~s}^{-1}$. The second assumption is justified by the small $\gamma\left(\sim 0.0056 \mathrm{~K}^{-1}\right)$ so that the variation of $s_{s}^{*}$ with radius can be neglected. This simplification allows a closed-form analytic solution, which facilitates the understanding of the dependence of intensity and structure on the outflow temperature profile (16). With $f=0$ and $\gamma=0$, (6) and (7) become

$$
V^{2}=\frac{C_{k}}{C_{d}}\left(T_{b}-T_{\infty}\right)\left(s_{s 0}^{*}-s^{*}\right),
$$

and

$$
\frac{d s^{*}}{d M}=-\frac{C_{k}}{C_{d}} \frac{\left(s_{s 0}^{*}-s^{*}\right)}{M},
$$

respectively, where by $(15), s_{s}^{*}=s_{s 0}^{*}$ for all radii.

Approximating (16) by a linear function gives

$$
T_{\infty}=T_{b}-A\left(s^{*}-s_{0}^{*}\right)
$$

\footnotetext{
${ }^{1}$ This $T_{b}$ value is the temperature on the sounding that has the minimum $s^{*}$. More details can be found in section 5c.
} 
where $A$ is a constant that determines the sounding slope and $s_{0}^{*}$ is the boundary layer-top saturation entropy at the outer radius $r_{0}$. Using (19) in (17),

$$
V^{2}=\frac{C_{k}}{C_{d}} A\left(s^{*}-s_{0}^{*}\right)\left(s_{s 0}^{*}-s^{*}\right) .
$$

To determine the maximum $V$, we consider $\partial V /\left.\partial s^{*}\right|_{s^{*}=s_{m}^{*}}=0$, to find

$$
s_{m}^{*}=\frac{1}{2}\left(s_{s 0}^{*}+s_{0}^{*}\right)
$$

Note that the subscript $m$ indicates the values at the radius of maximum wind for all parameters. Using (21) in (20),

$$
V_{m}=\frac{1}{2}\left(\frac{C_{k}}{C_{d}} A\right)^{1 / 2}\left(s_{s 0}^{*}-s_{0}^{*}\right) .
$$

Eq. (18) can be used to obtain $M$ as a function of $s^{*}$ by integration inward from the outer radius $r_{0}$ where $\left(M, s^{*}\right)=$ $\left(M_{0}, s_{0}^{*}\right)$, which gives

$$
\frac{M}{M_{0}}=\left(\frac{s_{s 0}^{*}-s^{*}}{s_{s 0}^{*}-s_{0}^{*}}\right)^{C_{d} / C_{k}} .
$$

From (21) and (23), we have

$$
M_{m}=M_{0}\left(\frac{1}{2}\right)^{C_{d} / C_{k}}
$$

using (22) and the definition of $M$ with $f=0$ then gives,

$$
r_{m}=\frac{M_{m}}{V_{m}}=\frac{M_{0}\left(\frac{1}{2}\right)^{C_{d} / C_{k}}}{\frac{1}{2}\left(\frac{C_{k}}{C_{d}}\right)^{1 / 2}\left(s_{s 0}^{*}-s_{0}^{*}\right)}(A)^{-1 / 2} .
$$

Dividing (20) by $V_{m}^{2}$ gives

$$
\frac{V^{2}}{V_{m}^{2}}=\frac{4\left(s^{*}-s_{0}^{*}\right)\left(s_{s 0}^{*}-s^{*}\right)}{\left(s_{s 0}^{*}-s_{0}^{*}\right)^{2}} .
$$

Eliminating $M_{0}$ between (23) and (24) allows the normalized $M$ to be expressed as

$$
\left(\frac{M}{M_{m}}\right)^{2}=\left(\frac{s_{s 0}^{*}-s^{*}}{s_{s 0}^{*}-s_{m}^{*}}\right)^{2 C_{d} / C_{k}}=\left(2 \frac{s_{s 0}^{*}-s^{*}}{s_{s 0}^{*}-s_{0}^{*}}\right)^{2 C_{d} / C_{k}},
$$

which can be solved for $s^{*}$ as

$$
s^{*}=s_{s 0}^{*}-\left(\frac{s_{s 0}^{*}-s_{0}^{*}}{2}\right)\left(\frac{r V}{r_{m} V_{m}}\right)^{C_{k} / C_{d}} .
$$

After setting $\tilde{V}=V / V_{m}, \tilde{r}=r / r_{m}$, using (28) in (26) and rearranging, we have

$$
\tilde{V}^{2}-2(\tilde{r} \tilde{V})^{C_{k} / C_{d}}+(\tilde{r} \tilde{V})^{2 C_{k} / C_{d}}=0,
$$

which gives the relation between $\tilde{V}$ and $\tilde{r}$; the special case $C_{k} / C_{d}=1$ gives $\tilde{V}=2 \tilde{r} /\left(1+\tilde{r}^{2}\right)$, which is the same as Eq. (36) of
ER11 with $f=0$. Note $\tilde{V}(\tilde{r})$ has no dependence on $A$ in this linear-sounding case; the ratio of $C_{k} / C_{d}$ can modify $\tilde{V}(\tilde{r})$ such that a smaller $C_{k} / C_{d}$ leads to a broader structure (Fig. 1a). This dependence on $C_{k} / C_{d}$ is consistent with the analytic solution Eq. (36) of ER11 (Fig. 1b). Though the normalized profile of $C_{k} / C_{d}=0.5$ from (29) is broader than the ER11 result, we present evidence below showing that more realistic forms of the outflow temperature sounding can have a strong effect on narrowing the theoretical $\tilde{V}(\tilde{r})$.

The present numerical solutions of the Lilly model with $C_{k} / C_{d}=1, f=0, \gamma=0$ and the linear dependence of outflow temperature on moist entropy, (19) (Fig. 2), agree with the analytic solution to within round-off error. With increasing $A$, the maximum tangential wind $V_{m}$ increases and the radius of maximum wind $r_{m}$ decreases (Figs. 2a,d) as dictated by (22) and (25). The normalized tangential wind profiles as a function of normalized radius (Fig. 2b) are independent of $A$ as expected from (29). The corresponding $s^{*}$ and $s_{s}^{*}$ in the normalized radius coordinate are the same for all $A$ values (Fig. 2e). It is consistent with (21) that $s_{m}^{*}$ is determined by the inputs of $s_{s 0}^{*}$ and $s_{0}^{*}$, while it has no dependence on $A$ (Fig. 2c). Under the assumed conditions, the air-sea disequilibrium decreases with decreasing radius (Fig. 2f).

\section{b. Linear $T_{\infty}\left(s^{*}\right)$ with $f=0$ and $\gamma \neq 0$}

If we keep $f=0$ and allow $\gamma \neq 0$, the sea surface saturation entropy should increase toward the center according to (15). If we assume a linear increase in

$$
s_{s}^{*}=s_{s 0}^{*}+\varepsilon\left(s^{*}-s_{0}^{*}\right),
$$

where $\varepsilon$ is a constant in the range 0 to 1 , then (6) becomes

$$
V^{2}=\frac{C_{k}}{C_{d}}\left(T_{b}-T_{\infty}\right)\left[s_{s 0}^{*}-s^{*}+\varepsilon\left(s^{*}-s_{0}^{*}\right)\right] .
$$

Using (19) in (31) and repeating the procedure of finding $s_{m}^{*}$ and $V_{m}$, we find

$$
s_{m}^{*}=\frac{\left(s_{s 0}^{*}+s_{0}^{*}-2 \varepsilon s_{0}^{*}\right)}{2} \frac{1}{(1-\varepsilon)},
$$

so that

$$
V_{m}=\frac{1}{2}\left(\frac{C_{k}}{C_{d}} A\right)^{1 / 2}\left(s_{s 0}^{*}-s_{0}^{*}\right)(1-\varepsilon)^{-1 / 2},
$$

which illustrates that allowing for an inward increase of $s_{s}^{*}$ produces a larger $V_{m}$ [cf. (33) and (22)]. Note that a constant $\varepsilon$ is assumed only for deriving an explicit analytic solution in (31) and is not necessary for the Lilly model in which $s_{s}^{*}$ is iteratively calculated by (15).

The numerical results from the Lilly model with $C_{k} / C_{d}=1$, $f=0, \gamma \neq 0$, and the linear dependence of outflow temperature on moist entropy (19) are shown in Fig. 3. In this case, the normalized radial profile of tangential wind varies with different $A$ such that the larger the $A$, the broader the radial 
(a) $\tilde{V}(\tilde{r})$ from (29)

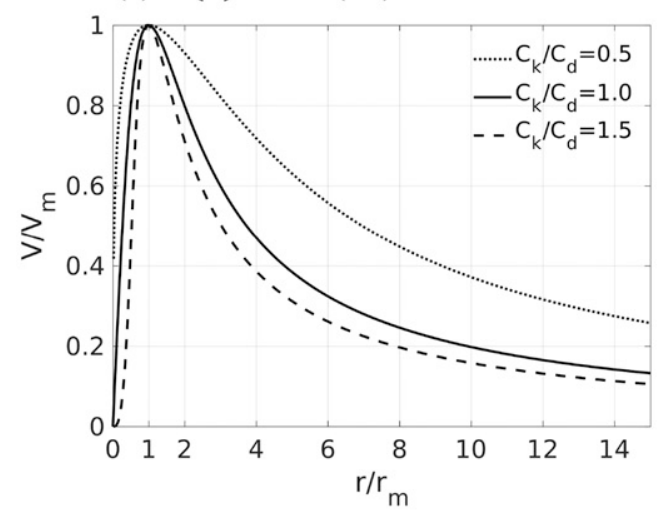

(c) $\tilde{V}(\tilde{r})$ from (44) with $\mathrm{B}=0.05 \mathrm{~K} \mathrm{~kg} \mathrm{~J}^{-1}$ (b) $\tilde{V}(\tilde{r})$ from (36) of ER 11

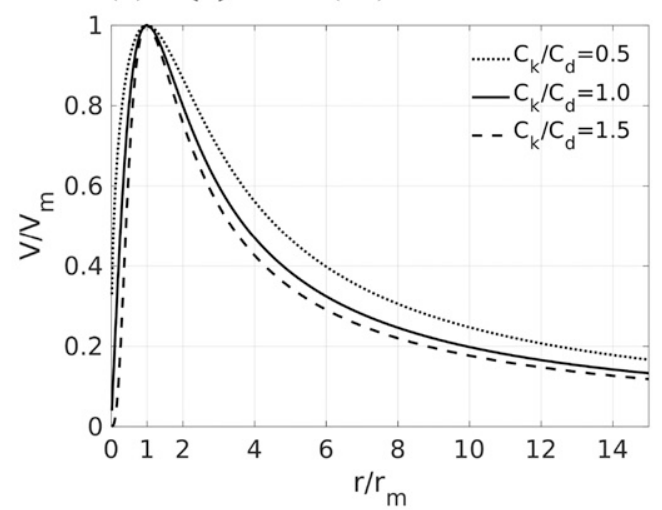

(d) $\tilde{V}(\tilde{r})$ from (44) with $\mathrm{B}=0.2 \mathrm{~K} \mathrm{~kg} \mathrm{~J}^{-1}$
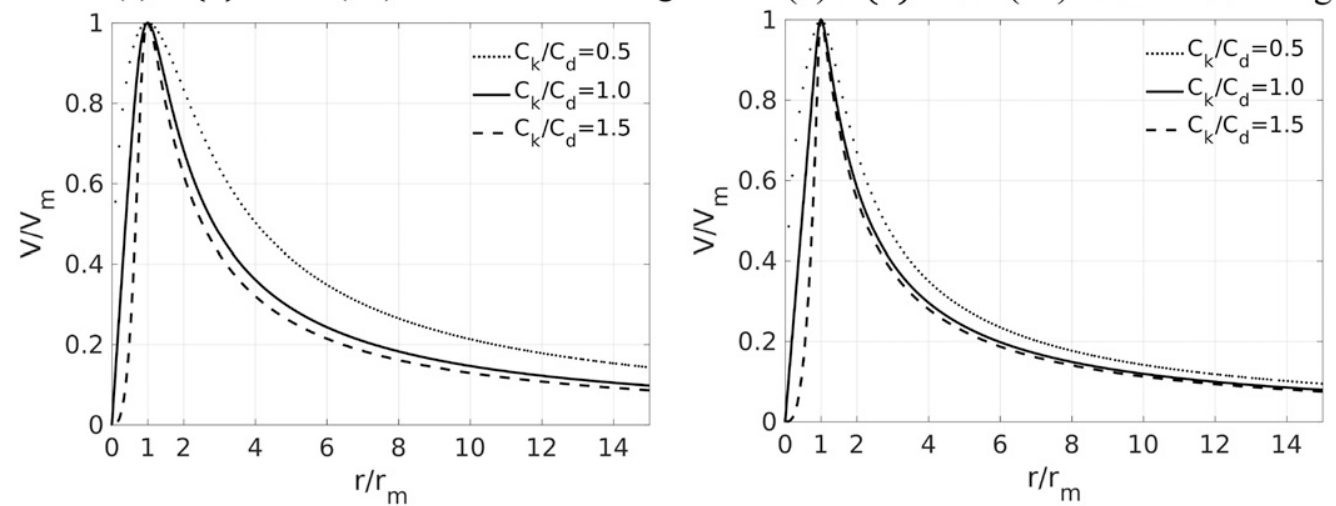

(e) $\tilde{V}(\tilde{r})$ from (44) with $\mathrm{B}=50 \mathrm{~K} \mathrm{~kg} \mathrm{~J}^{-1}$

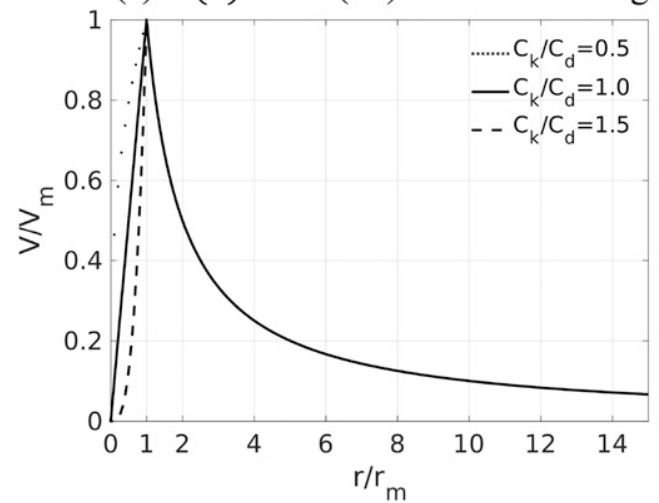

(f) $\tilde{V}(\tilde{r})$ from (46) and (49)

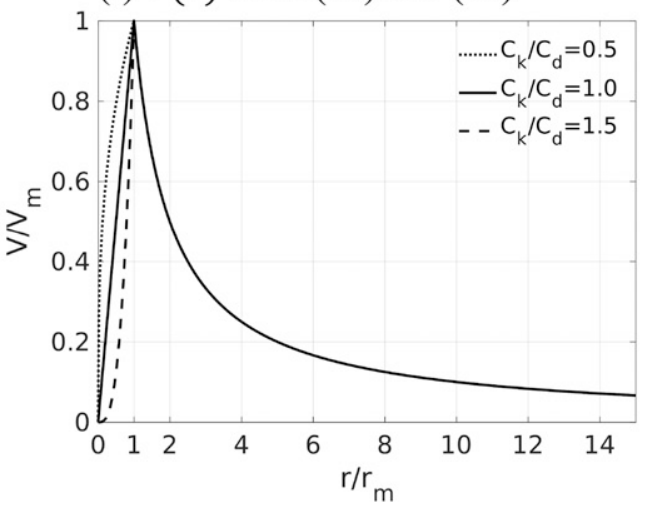

FIG. 1. Normalized tangential winds plotted against the normalized radius for the (a) analytic solution from (29), (b) analytic solution from (36) of ER11 with $f=0$, (c) solution from (44) with $B=0.05 \mathrm{~K} \mathrm{~kg} \mathrm{~J}^{-1}$, (d) solution from (44) with $B=0.2 \mathrm{~K} \mathrm{~kg} \mathrm{~J}^{-1}$, (e) solution from (44) with $B=50 \mathrm{~K} \mathrm{~kg} \mathrm{~J}^{-1}$, and (f) analytical solution from (46) and (49). Dotted lines are for $C_{k} / C_{d}=0.5$, solid lines are for $C_{k} / C_{d}=1.0$, and dashed lines are for $C_{k} / C_{d}=1.5$.

profile (Figs. 3a,b). Given the same $A$, the outflow temperatures at $M_{m}$ are much lower in Fig. 3c than those in Fig. 2c. Meanwhile, the $V_{m}\left(r_{m}\right)$ values in Fig. 3d are larger (smaller) than those shown in Fig. 2d. Consistent with (15), $s_{s}^{*}$ increases with decreasing radius and the rate of increase is greater with larger $A$ (Fig. 3e). However, because the increase rate of $s^{*}$ is larger than that of $s_{s}^{*}$, the air-sea disequilibrium still decreases with decreasing radius (Fig. 3f). c. Exponential $T_{\infty}\left(s^{*}\right)$ with $f=0$ and $\gamma=0$

In simulated TC outflows (see section 5), $s^{*}$ increases with height near the tropopause, while the temperature tends to a constant, which indicates the inadequacy of the linear function (19) near the tropopause. Following L86, we examined an exponential sounding $T_{\infty}\left(s^{*}\right)$ that has a tropopause (Fig. $4 \mathrm{c}$ ):

$$
T_{\infty}=T_{t}+\Delta T \times \exp \left[-B\left(s^{*}-s_{0}^{*}\right)\right]
$$



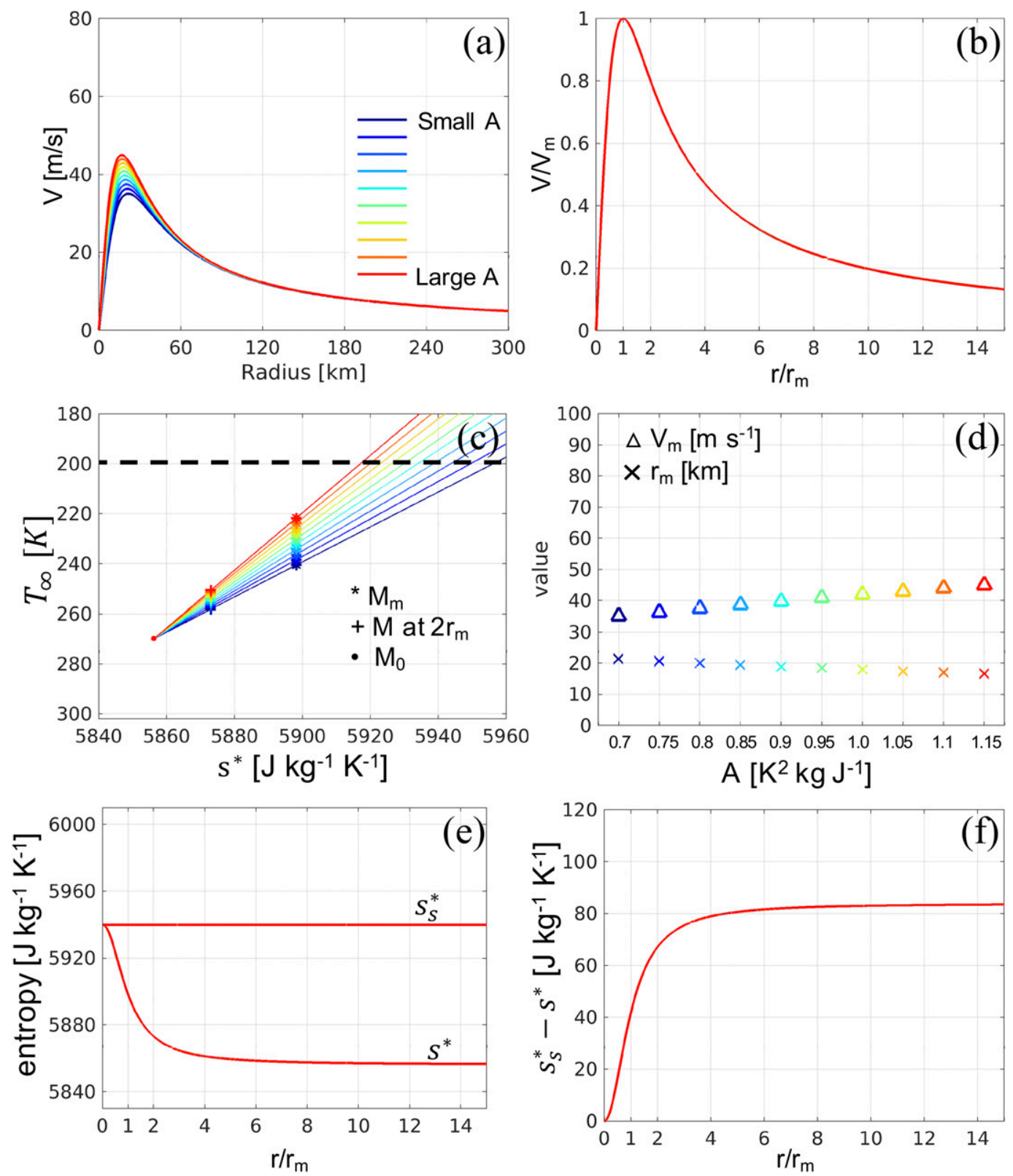

FIG. 2. Lilly model results using the linear function $T_{\infty}\left(s^{*}\right)=T_{b}-A\left(s^{*}-s_{0}^{*}\right)$ with $f=0$ and $\gamma=0, A=[0.7,0.75$, $0.8,0.85,0.9,0.95,1.0,1.05,1.1,1.15] \mathrm{K}^{2} \mathrm{~kg} \mathrm{~J}^{-1}$ for the (a) radial profiles of the tangential winds and (b) normalized tangential winds plotted against the normalized radius. Also shown are the (c) input function $T_{\infty}\left(s^{*}\right)$ in colors, while the dashed black line indicates $200 \mathrm{~K}$, the star denotes where the $M_{m}$ surface intersects with the sounding, the plus sign indicates the intersection of the $M$ surface across $2 r_{m}$ and the sounding, and the dot is where the $M_{0}$ surface intersects with the sounding; (d) maximum tangential wind $V_{m}$ and radius $r_{m}$ plotted against $A$; (e) profiles of $s_{s}^{*}$ and $s^{*}$; and (f) profiles of the air-sea saturation entropy difference as a function of the normalized radius.

where $T_{t}$ is the tropopause temperature $\left(T_{t}=199.19 \mathrm{~K}\right), \Delta T=$ $T_{b}-T_{t}$ and $B$ is a constant for sounding shape. Figure $4 \mathrm{c}$ shows that (34) gives a moist neutral sounding in the troposphere as $B \rightarrow \infty$. The Jordan hurricane season sounding is shown by the black line of Fig. $4 \mathrm{c}$, which is close to the case with $B=$ $0.05 \mathrm{~K} \mathrm{~kg} \mathrm{~J}^{-1}$.

Using (34) in (17), we have

$$
V^{2}=\frac{C_{k}}{C_{d}} \Delta T \times\left\{1-\exp \left[-B\left(s^{*}-s_{0}^{*}\right)\right]\right\}\left(s_{s 0}^{*}-s^{*}\right) .
$$

Repeating the procedure for finding $s_{m}^{*}$, we obtain

$$
V_{m}^{2}=\frac{C_{k}}{C_{d}} \Delta T \times\left\{1-\exp \left(-B\left(s_{m}^{*}-s_{0}^{*}\right)\right)\right\}\left(s_{s 0}^{*}-s_{m}^{*}\right),
$$



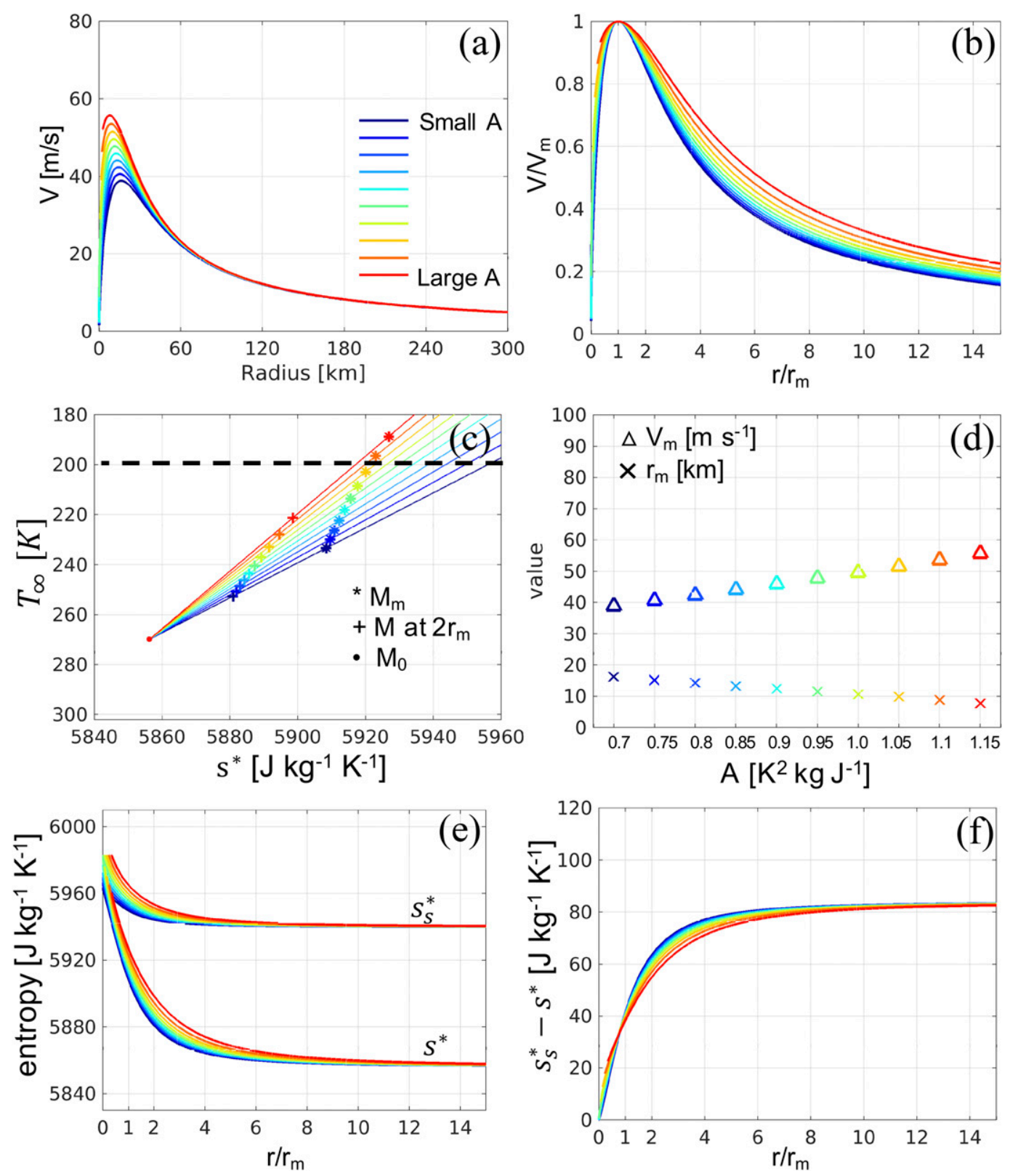

FIG. 3. As in Fig. 2, but for $\gamma \neq 0$.

where $s_{m}^{*}$ satisfies

$$
1-\left[1+B\left(s_{s 0}^{*}-s_{m}^{*}\right)\right] \exp \left[-B\left(s_{m}^{*}-s_{0}^{*}\right)\right]=0 .
$$

Here $s_{m}^{*}$ is obtained through solving (37) numerically. Since (23) does not rely on the outflow temperature, it can be used to obtain

$$
r_{m}=\frac{M_{m}}{V_{m}}=\frac{M_{0}}{V_{m}}\left(\frac{s_{s 0}^{*}-s_{m}^{*}}{s_{s 0}^{*}-s_{0}^{*}}\right)^{C_{d} / C_{k}} .
$$

Figure 5 shows the numerical solutions of $V_{m}$ and $r_{m}$ as a function of $B$ using the default values of $M_{0}, s_{0}^{*}$, and $s_{s 0}^{*}$ provided at the beginning of section 3 . With increasing $B, V_{m}$ increases monotonically and asymptotes to a value of $77 \mathrm{~m} \mathrm{~s}^{-1}$, while $r_{m}$ decreases first to around $18.28 \mathrm{~km}$ and then slowly asymptotes to a value of $19.48 \mathrm{~km}$. Figure 5 also shows that $s_{m}^{*}$ decreases with increasing $B$ and asymptotes to $s_{0}^{*}$.

The relation among $B$ and the other variables $\left(V_{m}\right.$ and $\left.r_{m}\right)$ in the extreme cases with $B \rightarrow 0$ and $B \rightarrow \infty$ can be obtained analytically. For $B \rightarrow 0$, (35) becomes

$$
\begin{aligned}
V^{2} & \cong \frac{C_{k}}{C_{d}} \Delta T \times\left\{1-\left[1-B\left(s^{*}-s_{0}^{*}\right)\right]\right\}\left(s_{s 0}^{*}-s^{*}\right) \\
& =\frac{C_{k}}{C_{d}} \Delta T \times B\left(s^{*}-s_{0}^{*}\right)\left(s_{s 0}^{*}-s^{*}\right),
\end{aligned}
$$



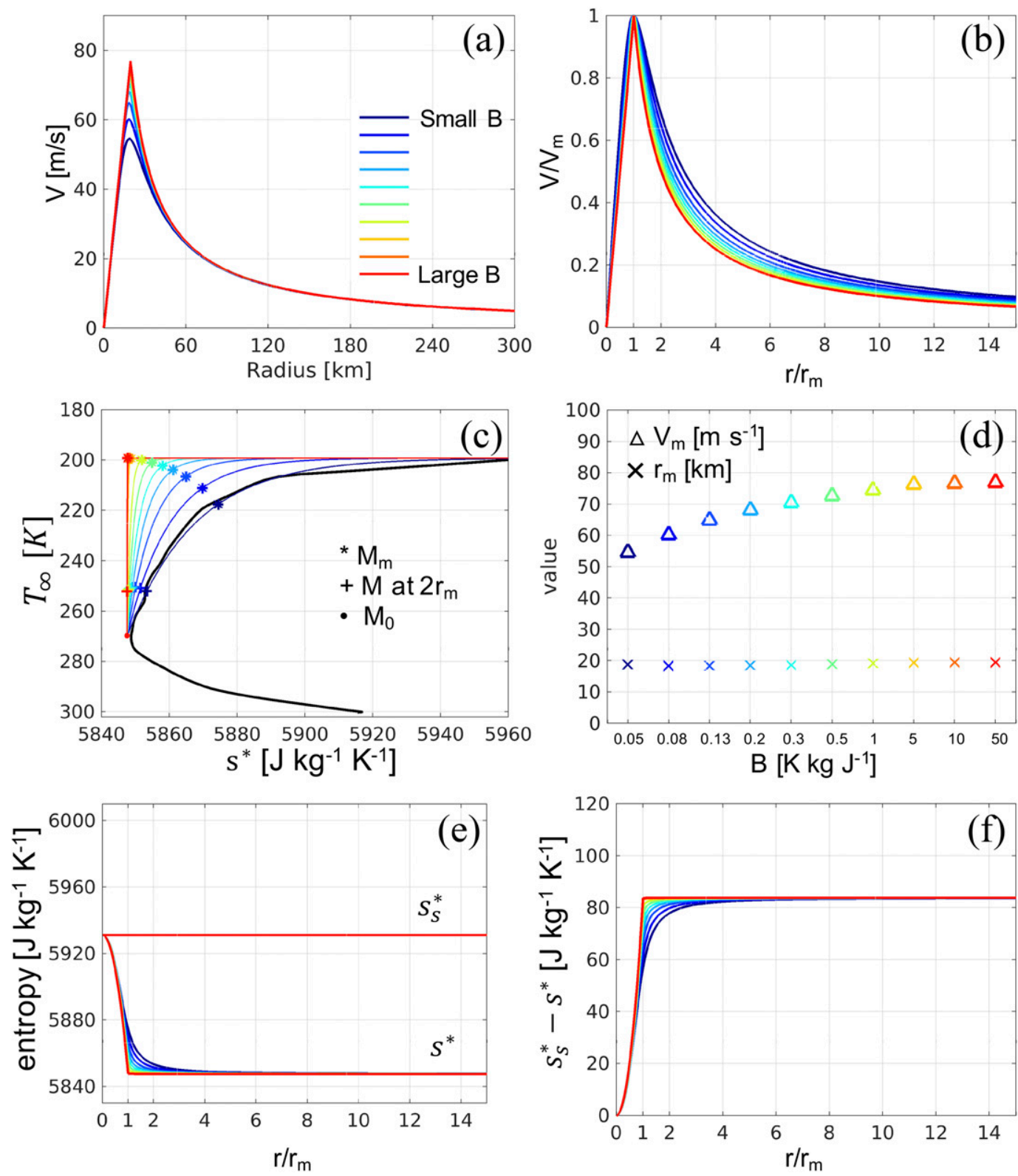

FIG. 4. As in Fig. 2, but using the exponential function $T_{\infty}=T_{t}+\Delta T \times \exp \left[-B\left(s^{*}-s_{0}^{*}\right)\right]$ with $f=0$ and $\gamma=0$, $B=[0.05,0.08,0.13,0.2,0.3,0.5,1.0,5.0,10.0,50.0] \mathrm{K} \mathrm{kg} \mathrm{J}^{-1}$. The black line in (c) is the Jordan hurricane season sounding.

which is exactly the same as (20) with $\Delta T \times B$ replacing $A$, and therefore (22) and (25) give, respectively, $V_{m} \cong$ $(1 / 2)\left[\left(C_{k} / C_{d}\right) \Delta T \times B\right]^{1 / 2}\left(s_{s 0}^{*}-s_{0}^{*}\right)$ and $r_{m} \cong\left\{\left[M_{0}(1 / 2)^{C_{d} / C_{k}}\right] /\right.$ $\left.\left[(1 / 2)\left(C_{k} / C_{d}\right)^{1 / 2}\left(s_{s 0}^{*}-s_{0}^{*}\right)\right]\right\}(\Delta T \times B)^{-1 / 2}$. As shown in Fig. 5, for small $B\left(<0.1 \mathrm{~K} \mathrm{~kg} \mathrm{~J}^{-1}\right), V_{m}$ increases with increasing $B$, while $r_{m}$ has the opposite tendency. For $B \rightarrow \infty$, we define $\delta s^{*} \equiv s_{m}^{*}-s_{0}^{*}$ and $\Delta s^{*} \equiv s_{s 0}^{*}-s_{0}^{*}$, and assume $\delta s^{*} \ll \Delta s^{*}$ (which will be verified below), so that (37) becomes

$$
\exp \left(B \delta s^{*}\right) \cong 1+B \Delta s^{*} \cong B \Delta s^{*},
$$

which gives

$$
\delta s^{*} \cong \ln \left(B \Delta s^{*}\right) / B .
$$

With $\lim \left(\delta s^{*} / \Delta s^{*}\right) \cong \lim \left[\ln \left(B \Delta s^{*}\right) / B \Delta s^{*}\right] \rightarrow 0$, we find that $\delta s^{*} \ll \Delta s^{*}$ as assumed. This latter inequality can also be seen from Fig. 5 for the sounding with $B=50 \mathrm{~K} \mathrm{~kg} \mathrm{~J}^{-1}$, which has $\delta s^{*} \sim 0.167 \mathrm{~J} \mathrm{~kg}^{-1} \mathrm{~K}^{-1}$, which is much smaller than $\Delta s^{*} \sim$ $83.87 \mathrm{~J} \mathrm{~kg}^{-1} \mathrm{~K}^{-1}$. With (41) and $\delta s^{*} \ll \Delta s^{*}$, (36) becomes $V_{m} \cong$ $\left[\left(C_{k} / C_{d}\right) \Delta T \times \Delta s^{*}\right]^{1 / 2}$, while (38) becomes $r_{m} \cong\left(C_{d} / C_{k}\right)^{1 / 2}\left[M_{0} /\right.$ $\left(\Delta T \times \Delta s^{*}\right)^{1 / 2}$ ]. These solutions with $B \rightarrow \infty$ show that both 


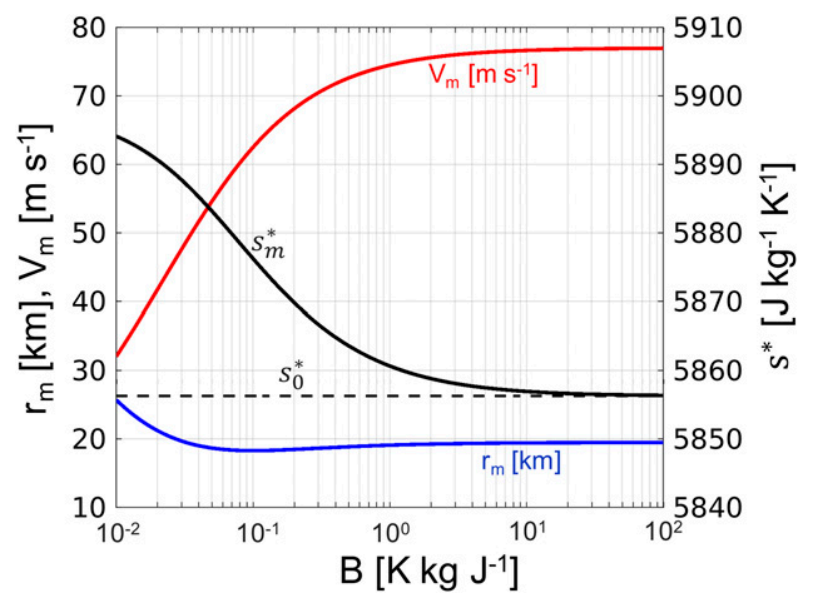

FIG. 5. Analytic solutions of $s_{m}^{*}$ (black), $V_{m}$ (red), and $r_{m}$ (blue) as a function of $B$ from Eqs. (36)-(38) using the default inputs. $V_{m}$ and $r_{m}$ share the same $y$ axis on the left, while $s_{m}^{*}$ and $s_{0}^{*}$ share the same $y$ axis on the right.

$V_{m}$ and $r_{m}$ asymptotically approach constants, which is consistent with Fig. 5 in the region where $B$ is large $\left(>30 \mathrm{~K} \mathrm{~kg} \mathrm{~J}^{-1}\right)$.

After solving for $s_{m}^{*}$, the radial structure of the tangential wind can also be determined. From (23), we have

$$
s^{*}=s_{s 0}^{*}-\left(s_{s 0}^{*}-s_{m}^{*}\right)\left(\frac{M}{M_{m}}\right)^{C_{k} / C_{d}}
$$

Dividing (35) by (36) and using (38), we obtain

$$
\frac{V^{2}}{V_{m}^{2}}=\frac{1-\exp \left[-B\left(s^{*}-s_{0}^{*}\right)\right]}{1-\exp \left[-B\left(s_{m}^{*}-s_{0}^{*}\right)\right]}\left(\frac{M}{M_{m}}\right)^{C_{k} / C_{d}} .
$$

Setting $\tilde{V}=V / V_{m}$ and $\tilde{r}=r / r_{m}$ and replacing $s^{*}$ with (42), the relation between $\tilde{V}$ and $\tilde{r}$ can be expressed by

$$
\tilde{V}^{2}=\frac{1-\exp \left\{-B\left[\left(s_{s 0}^{*}-s_{0}^{*}\right)-\left(s_{s 0}^{*}-s_{m}^{*}\right)(\tilde{r} \tilde{V})^{C_{k} / C_{d}}\right]\right\}}{1-\exp \left[-B\left(s_{m}^{*}-s_{0}^{*}\right)\right]}(\tilde{r} \tilde{V})^{C_{k} / C_{d}},
$$

with $s_{m}^{*}$ known through solving (37). Profiles of $\tilde{V}(\tilde{r})$ are shown with $B=0.05 \mathrm{~K} \mathrm{~kg} \mathrm{~J}^{-1}$ and $B=0.2 \mathrm{~K} \mathrm{~kg} \mathrm{~J}^{-1}$ in Figs. $1 \mathrm{c}$ and $1 \mathrm{~d}$, respectively. Though the shape of the exponential sounding ( $B$ parameter) can modify the normalized tangential wind profiles for all $C_{k} / C_{d}$, the dependence of the normalized tangential wind profiles on $C_{k} / C_{d}$ under different $B$ values (Figs. 1c,d) still has the same tendency as the ER11 model (Fig. 1b). This indicates a universal dependency of the normalized tangential wind profiles on $C_{k} / C_{d}$ such that a smaller $C_{k} / C_{d}$ leads to a broader vortex regardless of the sounding shape except for close-to-neutral soundings (Fig. 1e). In the limit of large $B$, the profiles shown in Fig. 1e can be derived analytically. Using (41) and $\delta s^{*} \ll \Delta s^{*}$, (44) becomes

$$
\begin{aligned}
\tilde{V}^{2} & =\frac{1-\exp \left\{-B\left[\Delta s^{*}-\left(\Delta s^{*}-\delta s^{*}\right)(\tilde{r} \tilde{V})^{C_{k} / C_{d}}\right]\right\}}{1-\exp \left(-B \delta s^{*}\right)}(\tilde{r} \tilde{V})^{C_{k} / C_{d}} \\
& \cong\left(1-\exp \left\{-B \Delta s^{*}\left[1-(\tilde{r} \tilde{V})^{C_{k} / C_{d}}\right]\right\}\right)(\tilde{r} \tilde{V})^{C_{k} / C_{d}}
\end{aligned}
$$

For $\tilde{r} \tilde{V}<1$, the exponential term is small compared to unity and (45) becomes $\tilde{V}^{2} \cong(\tilde{r} \tilde{V})^{C_{k} / C_{d}}$ so that

$$
\tilde{V} \cong(\tilde{r})^{\left(C_{k} / C_{d}\right) /\left(2-C_{k} / C_{d}\right)},
$$

which is the normalized wind profile inside $r_{m}(\tilde{r}<1)$.

For $\tilde{r} \tilde{V} \sim 1$, the term of $-B \Delta s^{*}\left[1-(\tilde{r} \tilde{V})^{C_{k} / C_{d}}\right]$ is small enough that the approximation $e^{x} \cong 1+x$ can be used in (45) to obtain

$$
\tilde{V}^{2} \cong B \Delta s^{*}\left[1-(\tilde{r} \tilde{V})^{C_{k} / C_{d}}\right](\tilde{r} \tilde{V})^{C_{k} / C_{d}} .
$$

Rearranging (47) to obtain

$$
\tilde{V}^{C_{k} / C_{d}} \cong \tilde{r}^{-C_{k} / C_{d}}-\frac{\tilde{V}^{\left(2-C_{k} / C_{d}\right)}}{B \Delta s^{*} \tilde{r}^{\left(2 C_{k} / C_{d}\right)}},
$$

one sees that, for $B \Delta s^{*} \gg 1$, the second term on the right-hand side of (48) is very small so that (48) becomes

$$
\tilde{V} \cong \tilde{r}^{-1}
$$

which gives the normalized wind profile outside $r_{m}(\tilde{r}>1)$. Note that (49) has no dependence on $C_{k} / C_{d}$. The profiles of $\tilde{V}(\tilde{r})$ from (44) with $B=50 \mathrm{~K} \mathrm{~kg} \mathrm{~J}^{-1}$ in Fig. 1e are almost identical to the approximate solution (46) and (49) in Fig. 1f, which confirms that the approximations in (45)-(49) are valid for close-to-neutral soundings.

Though the sensitivities of $\tilde{V}(\tilde{r})$ to $B$ for intermediate values cannot be directly seen through the complicated expressions of (37) and (44), the numerical results from the Lilly model with $C_{k} / C_{d}=1, f=0, \gamma=0$ and varying exponential profiles (34) are presented in Fig. 4. Using the exponential function (34) allows the existence of a tropopause and a continuous $s^{*}$ (Fig. 4c). As shown in Fig. 5, the dependence of $V_{m}$ on $B$ has the same trend as solutions for the linear outflow temperature sounding (19) in that $V_{m} \propto B$ for small $B$; however, $r_{m}$ is almost independent of $B$ (Fig. $4 \mathrm{~d}$ ). Unlike the solutions for a linear outflow temperature sounding (Figs. 2e,f), Figs. 4e and 4f show that $s^{*}$ in the normalized-radius coordinate changes with $B$ such that the air-sea disequilibrium increases with increasing $B$ and the normalized tangential wind profiles (Fig. 4b) for $r / r_{m}>1$ narrow to the asymptotic limit given by (49).

Given that the analytic solutions for the exponential sounding case with $f=0$ and $\gamma=0$ are already complicated enough, we will use only numerical solutions of the Lilly model to investigate the more realistic cases with $f \neq 0$ and $\gamma \neq 0$ in section 4 .

\section{Steady-state TC structure and intensity sensitivities to the outflow-temperature profiles and boundary conditions}

In this section, we will investigate the solution sensitivities of the Lilly model by varying one parameter at a time using the exponential outflow-temperature profile (34). Though the changes of the parameters are most likely correlated (e.g., a change of sea surface temperature should change the boundary layer and tropopause temperatures), changing each parameter separately is helpful for understanding the role of each in TC structure and intensity. The sensitivities tested here are based 

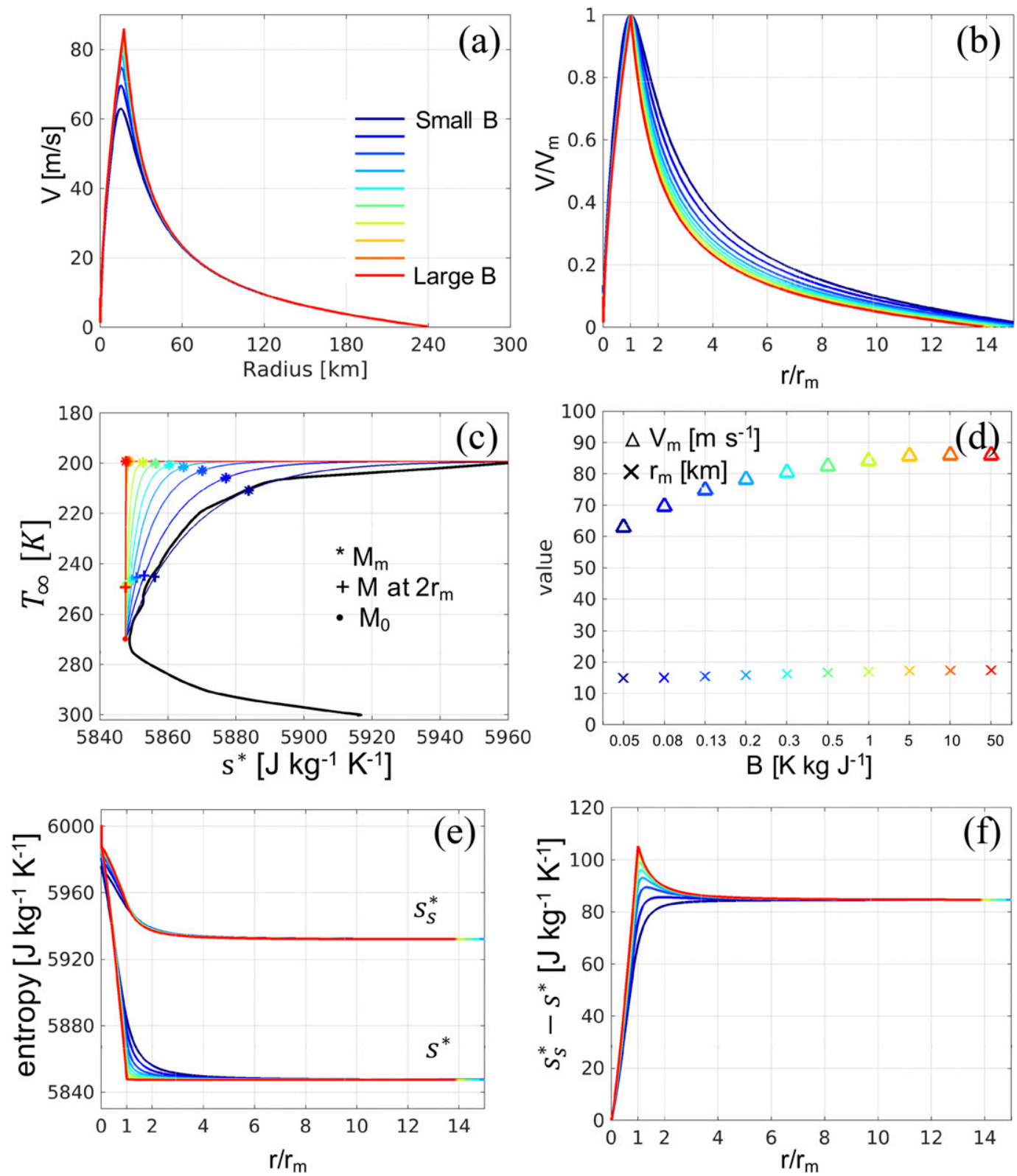

FIG. 6. As in Fig. 4, but for $f \neq 0$ and $\gamma \neq 0$.

on a TC at steady state; the influence of these parameters during the development stage is not considered.

The basic parameter values are $T_{b}=269.87 \mathrm{~K}, T_{t}=199.19 \mathrm{~K}$, $s_{0}^{*}=5856.26 \mathrm{~J} \mathrm{~kg}^{-1} \mathrm{~K}^{-1}, s_{s 0}^{*}=5940.13 \mathrm{~J} \mathrm{~kg}^{-1} \mathrm{~K}^{-1}, M_{0}=1.5 \times$ $10^{6} \mathrm{~m}^{2} \mathrm{~s}^{-1}, f=5 \times 10^{-5} \mathrm{~s}^{-1}, \gamma=0.0056 \mathrm{~K}^{-1}$, and $B=$ $0.05 \mathrm{~K} \mathrm{~kg} \mathrm{~J}^{-1}$. These values are used as default setups in this section except for the varying parameter specifically pointed out for the purpose of testing its sensitivity.

\section{a. Solution sensitivity to exponential sounding profiles}

To test the sensitivity of exponential sounding profiles, the sounding shape parameter $B$ is varied. Figure 6 shows the numerical solutions of the Lilly model using the exponential profile (34) with $f \neq 0$ and $\gamma \neq 0$. In comparison to Fig. 4 , including the Coriolis effect changes the tangential wind profile at the outer radius and makes the tangential wind vanish at a radius of $240 \mathrm{~km}$ for the given $M_{0}$ (Fig. 6a), while allowing $s_{s}^{*}$ to increase with decreasing radius mainly affects the inner-core tangential wind inside $3 r_{m}$ (cf. Figs. 4e, 6e). The air-sea disequilibrium can even increase with decreasing radius and maximizes near $r_{m}$ when $B$ is large enough (Fig. 6f). The normalized tangential wind profiles and the magnitudes of the maximum tangential wind (Figs. 6b,d) have a similar dependence on $B$ as in the case with $f=0$ and $\gamma=0$ (Figs. $4 \mathrm{~b}, \mathrm{~d}$ ). The profiles of $T_{\infty}\left(s^{*}\right)$ have a role in determining the normalized tangential wind profiles such 

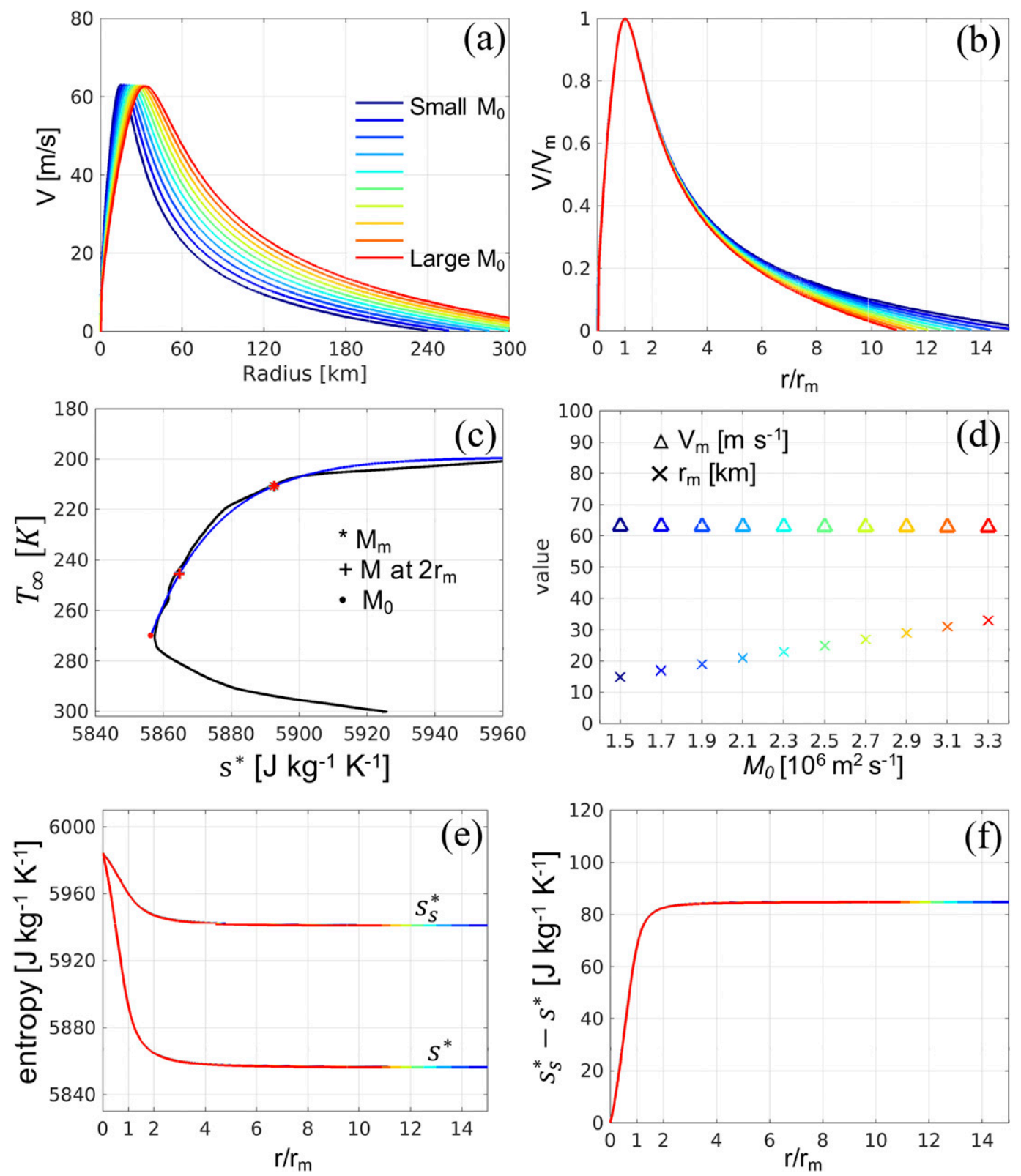

FIG. 7. As in Fig. 6, but for the solution sensitivity to $M_{0}$ in the Lilly model using the exponential function $T_{\infty}=T_{t}+\Delta T \times \exp \left[-0.05\left(s^{*}-s_{0}^{*}\right)\right], M_{0} \in[1.5,3.3] \times 10^{6} \mathrm{~m}^{2} \mathrm{~s}^{-1}$ with an interval of $0.2 \times 10^{6} \mathrm{~m}^{2} \mathrm{~s}^{-1}$.

that the smaller the $B$, the broader the vortex outside $r_{m}$ (Fig. 6b).

\section{b. Solution sensitivity to $M_{0}$}

The sensitivity of the solutions to $M_{0}$ is shown in Fig. 7. The different $M_{0}$ represents different TC sizes since it determines the outer radius $r_{0}$ through (6). The radial profiles of tangential winds in physical space vary with different $M_{0}$ at all radii (Fig. 7a); a larger $M_{0}$ represents a larger storm. The normalized tangential wind profiles share the same inner-core structure while the Coriolis effect on the outer-core wind profile makes the normalized structure differ (Fig. 7b). But according to section 2, the Lilly model is not applicable to these outer radii since the motion is downward and unsaturated there. The temperature difference of $\left(T_{b}-T_{\infty}\right)$ and the air-sea disequilibrium of $\left(s_{s}^{*}-s^{*}\right)$ do not vary with different $M_{0}$ in the normalized radius coordinate (Figs. 7c,e,f). As predicted by the classic maximum potential intensity (E86) theory, other things being equal, all the variations in $M_{m}$ are accounted for by $r_{m}$ (Fig. 7d).

\section{c. Solution sensitivity to $s_{s 0}^{*}$}

Figure 8 presents the sensitivity of the solutions to the saturation sea surface entropy $s_{s 0}^{*}$. The change of $d s_{s 0}^{*}$ 

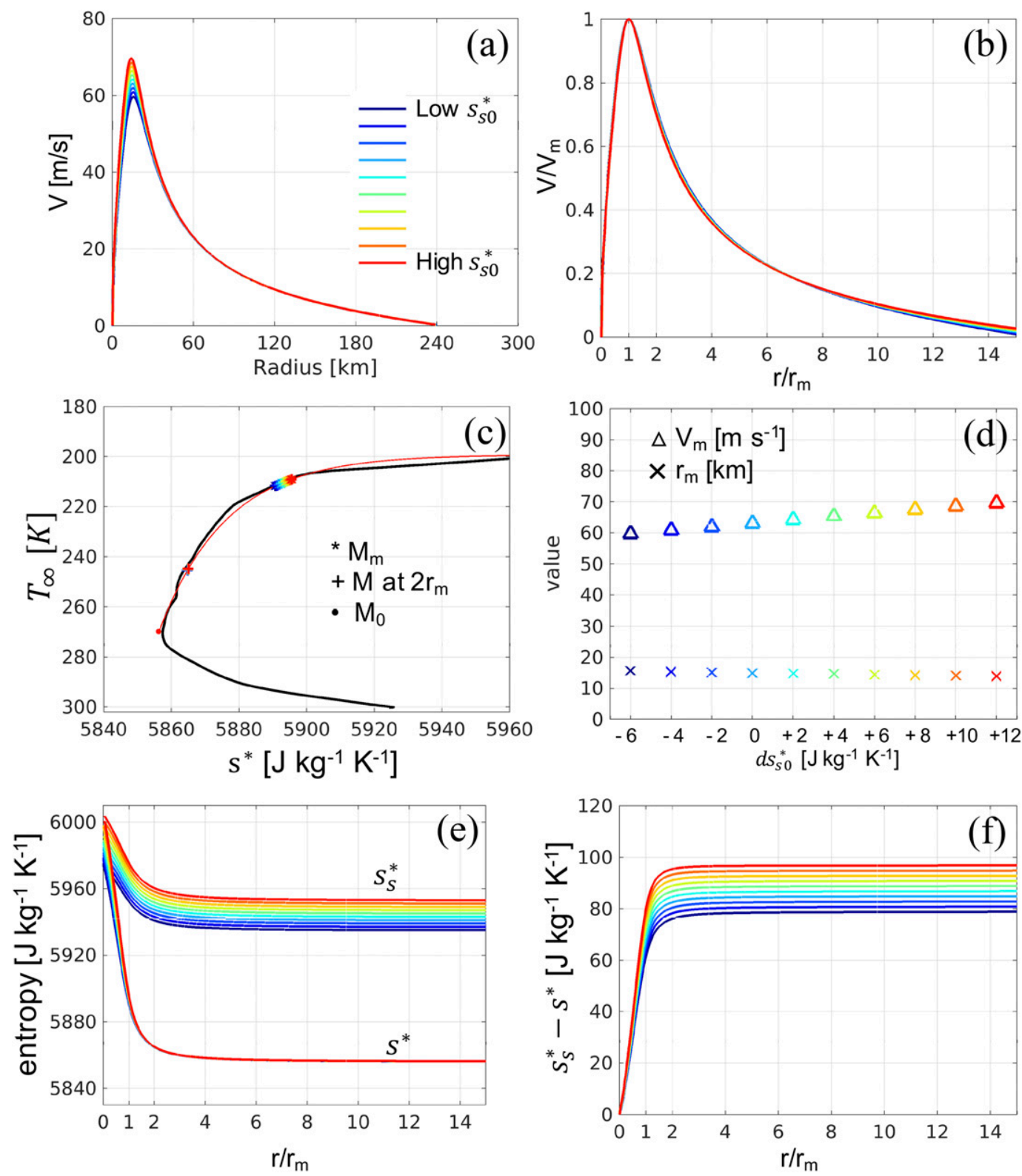

FIG. 8. As in Fig. 7, but for the solution sensitivity to $s_{s 0}^{*}, d s_{s 0}^{*} \in[-6,12] \mathrm{J} \mathrm{kg}^{-1} \mathrm{~K}^{-1}$ with an interval of $2 \mathrm{~J} \mathrm{~kg}^{-1} \mathrm{~K}^{-1}$.

indicates the deviation from the default value. Varying $s_{s 0}^{*}$ mainly changes the air-sea disequilibrium (Figs. 8e,f) and slightly changes the outflow temperature of $M_{m}$ (Fig. 8c). The tangential wind profile response is an increase near $r_{m}$ with increasing $s_{s 0}^{*}$ (Fig. 8a). Outside $2 r_{m}$, the difference of the air-sea disequilibrium is weakened by the small temperature difference $\left[\left(T_{b}-T_{\infty}\right) \sim O(1) \mathrm{K}\right]$ such that the tangential wind profiles exhibit negligible differences (Fig. 8a). The maximum intensity increases with rising sea surface temperature (Figs. 8a,d) as predicted by previous studies (Holland 1997; Emanuel 2005; Bengtsson et al. 2007). It is also seen that varying $s_{s 0}^{*}$ only slightly changes the normalized tangential wind profiles (Fig. 8b).

\section{d. Solution sensitivity to $T_{b}$ and $T_{t}$}

Similar to changing $s_{s 0}^{*}$, the sensitivities of the solutions to $T_{b}$ and $T_{t}$, respectively, are examined by varying $T_{b}$ and $T_{t}$ with $d T_{b}$ and $d T_{t}$ indicating the deviations from their default values. The sensitivity of the solutions to $T_{b}$ and $T_{t}$, respectively, lies in the tangential wind magnitude near $r_{m}$ (Figs. 9a,b). It is shown in Figs. $9 \mathrm{c}$ and $9 \mathrm{~d}$ that the temperature difference $\left(T_{b}-T_{\infty}\right)$ near $r_{m}$ increases with increasing $T_{b}$ or decreasing $T_{t}$, hence the tangential wind near $r_{m}$ increases with increasing $T_{b}$ or 

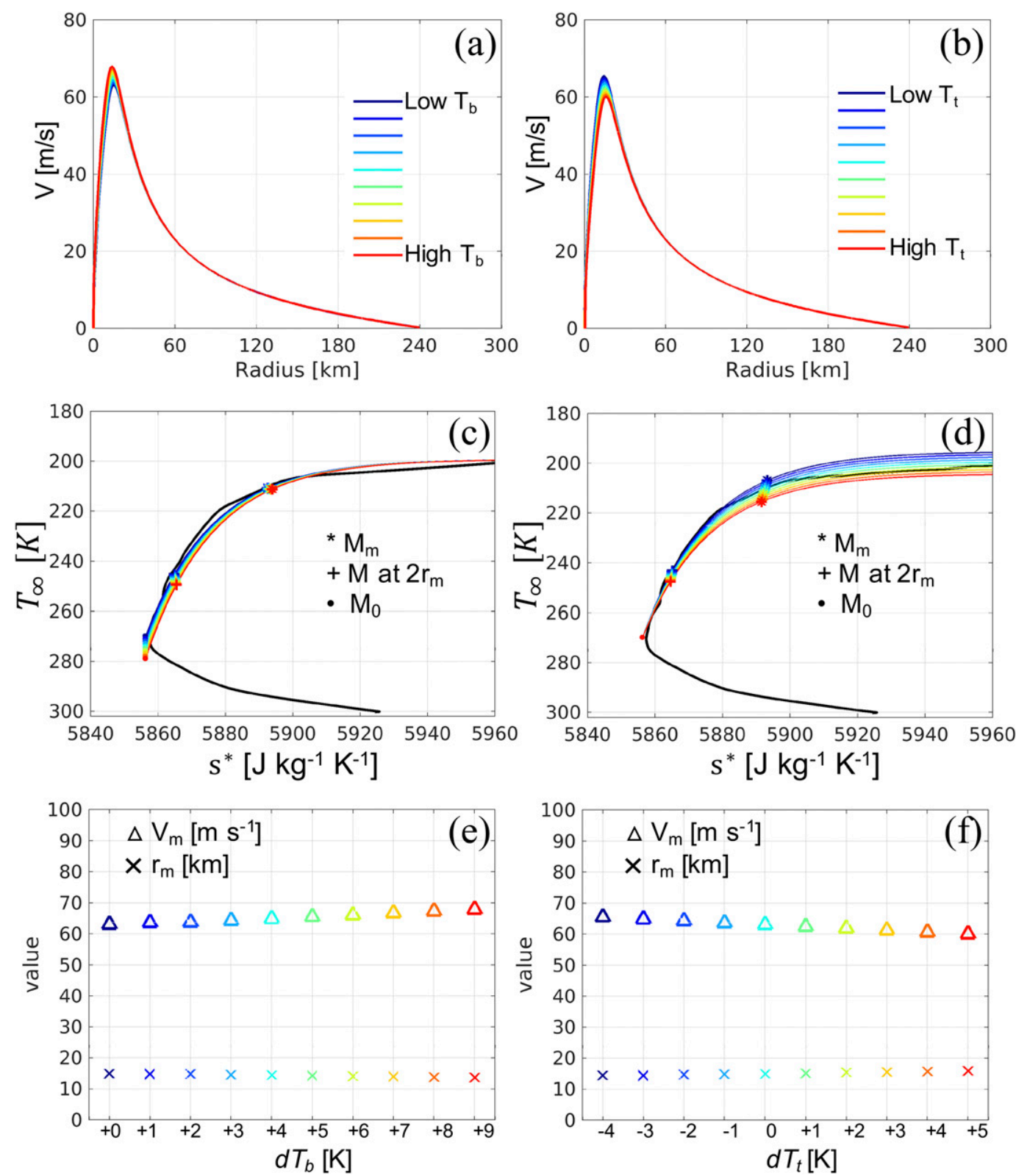

FIG. 9. Solution sensitivity to (left) $T_{b}$ and (right) $T_{t}$ in the Lilly model using the exponential function $T_{\infty}=T_{t}+\Delta T \times \exp \left[-0.05\left(s^{*}-s_{0}^{*}\right)\right]$. (left) $d T_{b} \in[0,9] \mathrm{K}$ with an interval of $1 \mathrm{~K}$. (right) $d T_{t} \in[-4,5] \mathrm{K}$ with an interval of $1 \mathrm{~K}$. Shown are (a),(b) the radial profiles of the tangential winds; (c),(d) the input function $T_{\infty}\left(s^{*}\right)$ in colors, while the black line indicates the Jordan hurricane season sounding, the star denotes where the $M_{m}$ surface intersects with the sounding, the plus indicates the intersection of the $M$ surface across $2 r_{m}$ and the sounding, and the dot is where the $M_{0}$ surface intersects with the sounding; (e) maximum tangential wind $V_{m}$ and radius $r_{m}$ plotted against $d T_{b}$; and (f) maximum tangential wind $V_{m}$ and radius $r_{m}$ plotted against $d T_{t}$.

decreasing $T_{t}$ (Figs. 9e,f) according to (6). A larger $T_{b}$ increases the temperature difference $\left(T_{b}-T_{\infty}\right)$ at all radii. However, $T_{\infty}$ changes with $T_{b}$ in the same way according to (34) and cancels most of the variation in $\left(T_{b}-T_{\infty}\right)$ due to $d T_{b}$ at the outer radii, which is the reason for the sensitivity in $V(r)$ only occurring near $r_{m}$. The insensitivity of outerradii wind to $T_{t}$ is because $T_{\infty}$ of the outer radii changes with $T_{t}$ to a much lesser extent, which slightly increases $\left(T_{b}-T_{\infty}\right)$. But at the same time, $s^{*}$ changes in a way to cancel the effect due to the $T_{\infty}$ change (Fig. 9d) such that the wind remains nearly unchanged.

Similar to the $s_{s 0}^{*}$ sensitivity results, the corresponding normalized wind profiles (not shown) are only slightly different from each other. Note that the $M_{m}$ surface actually cannot 
reach the tropopause temperature in exponential soundings, but the outflow temperature to which $M_{m}$ asymptotically approaches is positively correlated to $T_{t}$ (Fig. 9d).

\section{Comparison of solutions between a full-physics model and the Lilly model}

In section 4, we explored the sensitivity of the steady-state tropical cyclone structure and intensity in the Lilly model using the exponential profile, (34). To test the applicability of the Lilly model to more realistic TC conditions, we will compare the solutions from a full-physics numerical model and the Lilly model in this section.

\section{a. Full-physics model setup}

The axisymmetric, nonhydrostatic Cloud Model, version 1 (CM1; Bryan and Rotunno 2009b), is used in this study to simulate quasi-realistic TCs. The domain size is $1500 \mathrm{~km}$ in radius with a stretched grid: 1-km grid spacing for $r<300 \mathrm{~km}$ and linearly stretched to $15-\mathrm{km}$ grid spacing at $r=1500 \mathrm{~km}$ for $r \geq 300 \mathrm{~km}$. The vertical grid length varies from 50 to $200 \mathrm{~m}$ for $z<5 \mathrm{~km}$ and is fixed at $200 \mathrm{~m}$ for $z \geq 5 \mathrm{~km}$. There are total of 140 vertical levels. The lowest model level is at $25 \mathrm{~m}$ above the surface and the highest model level is at $25 \mathrm{~km}$. The Coriolis parameter is a constant with $f=5 \times 10^{-5} \mathrm{~s}^{-1}$. The sea surface temperature is fixed at $28^{\circ} \mathrm{C}$, while the Jordan hurricane season sounding is used to initialize the simulations. Constant $C_{k}$ and $C_{d}=10^{-3}$ are applied. The vertical and horizontal turbulent mixing lengths are 100 and $1000 \mathrm{~m}$, respectively.

\section{b. Initial conditions and CM1 simulation results}

The initial radial profiles of surface tangential wind are shown in Fig. 10a, which are the same as those used in Tao et al. (2020). There are three different $r_{m}$ values $(60,90$, and $120 \mathrm{~km}$, respectively) and two different wind-skirt parameters $(\mathrm{Bs}=1.0$ and 0.75 , respectively, a smaller Bs produces a broader radial profile). The initial maximum surface wind is $20 \mathrm{~m} \mathrm{~s}^{-1}$ for all simulations, while the initial tangential winds all vanish at $r=$ $1500 \mathrm{~km}$. After normalization, the six wind profiles collapse to two discrete normalized profiles separated by the wind-skirt parameter Bs (Fig. 10b).

The evolution of maximum tangential wind at $z=1.55 \mathrm{~km}$ is shown in Fig. 11a. The smaller TCs develop faster than the larger TCs (Xu and Wang 2018), while the ending intensities of the smaller TCs are weaker than those of the larger TCs. The relation between the steady-state $r_{m}$ and $V_{m}$ was studied in Tao et al. (2020), which shows that the larger $r_{m}$ corresponding to a larger $V_{m}$ is due to the effect of turbulent mixing. The steadystate tangential wind structure is drawn from the mean of the last $24 \mathrm{~h}$ of each simulation (Fig. 11b). Though the steady-state radial profiles of the tangential winds (Fig. 11b) corresponding to the different initial profiles (Fig. 10a) differ, their normalized profiles approximately settle to one structure especially inside $4 r_{m}$ (Fig. 11c).

c. Comparison between the Lilly model and the CM1 results Since the Lilly model requires a thermodynamic sounding $T_{\infty}\left(s^{*}\right)$ such that $s^{*}$ monotonically increases with decreasing temperature, the height of the boundary layer top, defined by (a) Initial V(r) profiles

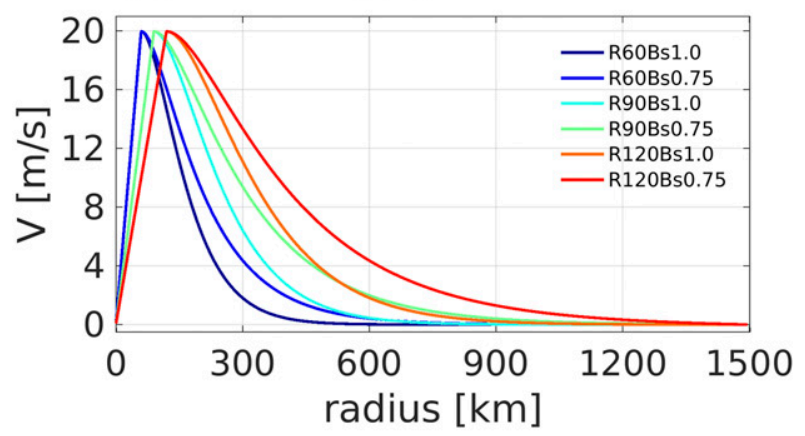

(b) Normalized V profiles

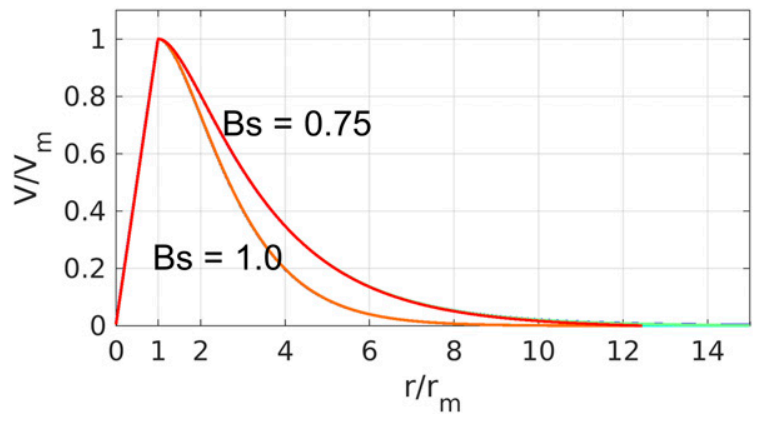

FIG. 10. (a) Radial profiles of the initial tangential winds used in CM1 simulations and (b) normalized initial tangential wind profiles plotted against the normalized radius. All profiles are from the lowest model level $(z=25 \mathrm{~m})$.

the minimum $s^{*}$, will be the top of the shallow convective layer in practice (E04). The disadvantage of this requirement is that the depth of the boundary layer (Fig. 12c) will be much higher than that from the traditional definition. Another point worth mentioning is the selection of the thermodynamic sounding location. L79 and L86 assumed that $T_{\infty}\left(s^{*}\right)$ can be taken from an unperturbed TC environmental sounding. However, in view of the discussion in section 2, we expect that the outflow temperature sounding evolves to be consistent with (2). The selected location of the sounding in ER11 is where the tangential wind vanishes in the outflow. Here we will adopt a similar strategy by choosing the sounding to be where the boundary layer-top tangential wind $V=0$ in the Lilly model, i.e., at $r_{0}=\sqrt{2 M_{0} / f}$, and proceed to solve for the Lilly model [(6), (7), (15), and (16)] starting from the minimum $s^{*}$ (Fig. 12c). Because $M$ does not change much outside $4 r_{m}$ in the Lilly model solutions (not shown), we select $M_{0}$ equal to $M$ at $r=4 r_{m}$ from the CM1 simulations. This choice also considers the fact that large-scale radiative subsidence dominates the outer-core tangential wind profiles (E04). Given the inputs of $s_{s 0}^{*}, s_{0}^{*}, \gamma$, and $T_{b}$ from the CM1 simulations at the outer radius $r_{0}=\sqrt{2 M_{0} / f}, M, s_{s}^{*}, r$, and $T_{\infty}$ are solved for iteratively from $r_{0}$ using the Lilly model.

Figure 12 shows a comparison between the Lilly model and CM1 solutions. Generally speaking, the Lilly model reproduces the CM1 $r_{m}$ but underestimates $V_{m}$ (Figs. 12a,d). Figure $12 \mathrm{~b}$ shows that the normalized tangential wind profiles from CM1 and the Lilly model match quite well in the region 

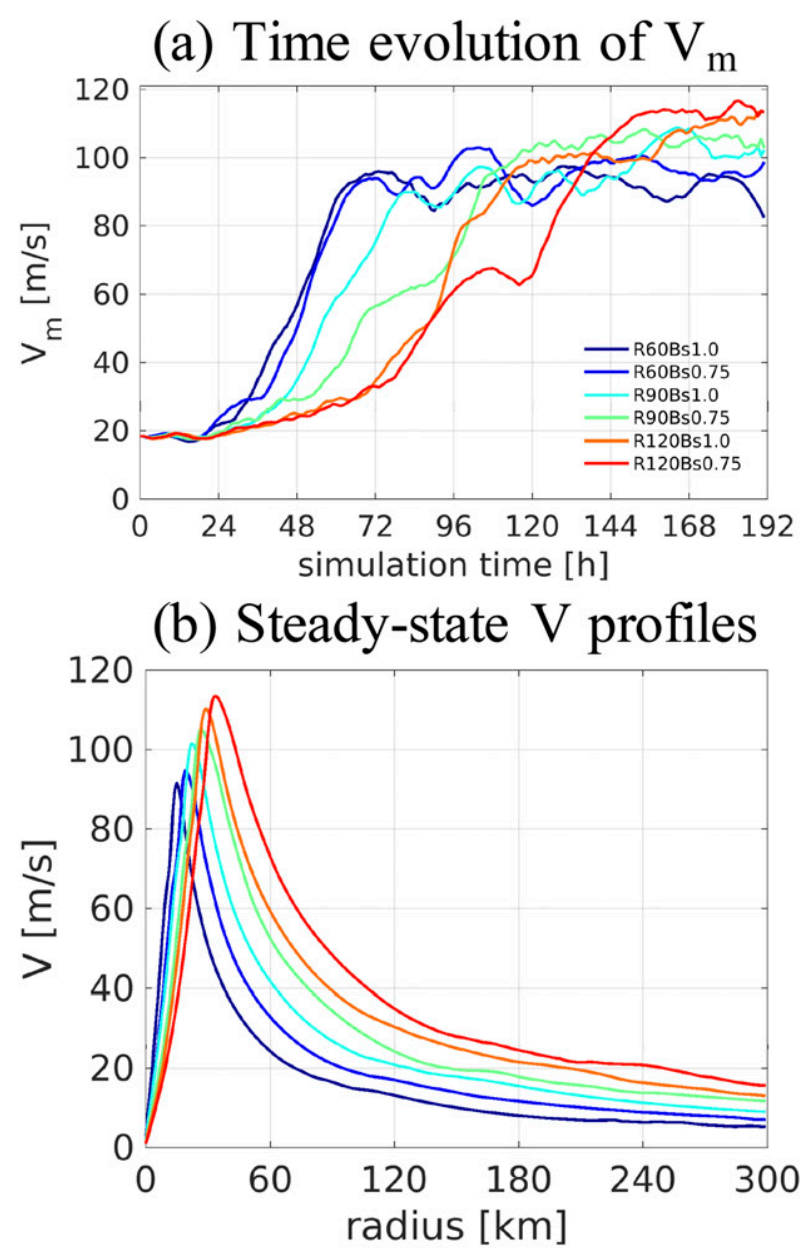

(c) Normalized V profiles

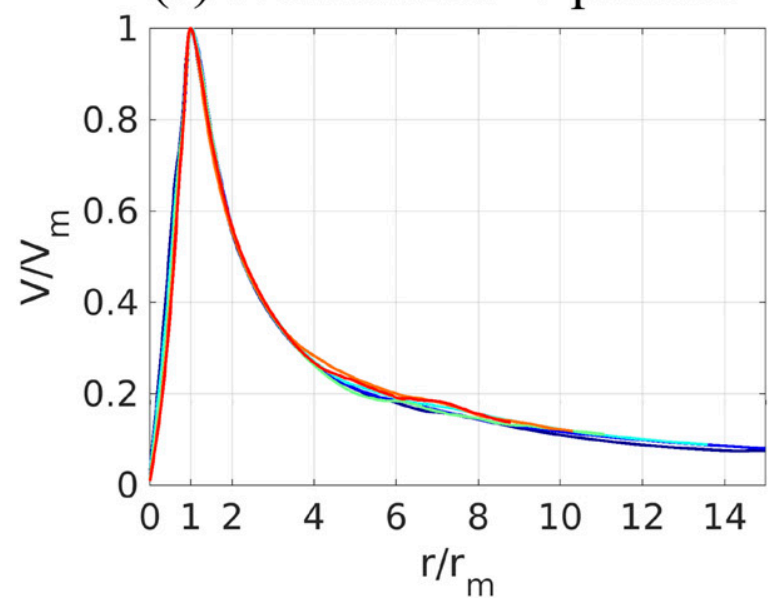

FIG. 11. (a) Time evolution of the maximum tangential winds at $z=1.55 \mathrm{~km}$, (b) the radial profiles of the tangential winds at $z=$ $1.55 \mathrm{~km}$ averaged from the last $24 \mathrm{~h}$ of the simulations (steady state), and (c) the normalized steady-state tangential winds plotted against the normalized radius at $z=1.55 \mathrm{~km}$. between $2 r_{m}$ and $4 r_{m}$, while inside $2 r_{m}$ and outside $4 r_{m}$, the Lilly model tends to produce weaker tangential winds. As discussed in section 2, the tangential wind profiles outside $4 r_{m}$ in real and simulated TCs should be controlled by radiative subsidence, while the Lilly model only applies in the inner core (inside $4 r_{m}$ ). In addition, the important processes of supergradient wind, turbulent mixing and evaporative cooling caused by strong downdrafts right outside the eyewall region are not included in the Lilly model, which cause discrepancies between the Lilly model and CM1 for tangential wind profiles inside $2 r_{m}$. The difference between the values of steady-state $V_{m}$ from CM1 and the Lilly model can vary from $16 \%$ (R60Bs1.0) to $35 \%$ (R120Bs0.75), which is consistent with the supergradient ratio discussed in Tao et al. (2020). Figure 12d shows that though the maximum tangential winds from the Lilly model are systematically weaker than the CM1 results, the radius of maximum wind is well captured by the Lilly model.

With the radial distribution of the boundary layer top $M$, $s_{s}^{*}$, and $s^{*}$ from the Lilly model, the thermodynamic sounding and the constraint from $M$ and $s^{*}$ conserved along streamlines, and using (6), we can obtain the radius and temperature on $M$ and $s^{*}$ surfaces above the boundary layer as well as the balanced portion of $M$ surfaces inside the boundary layer. The vertical cross sections of tangential winds, entropy and $M$ distributions in a temperature-radius coordinate are shown for the two extreme members (smallest storm: R60Bs1.0; largest storm: R120Bs0.75) from both models (Fig. 13). The temperature coordinate acts as a height coordinate since it monotonically decreases with height below the tropopause. The 20 and $30 \mathrm{~m} \mathrm{~s}^{-1}$ contours in these two cases are very similar between the CM1 results (left column of Fig. 13) and the Lilly model results (right column of Fig. 13). As seen in the CM1 results, the Lilly model results also exhibit a steeper $M_{m}$ in the R60Bs1.0 case than that in the R120Bs0.75 case. While the tangential winds of the Lilly model solutions near $M_{m}$ are all weaker than the CM1 results at all heights due to weaker intensity near $r_{m}$, it is quite clear that the Lilly model captures the size of the storm, the slope of the $M$ surfaces as well as the outflow temperature to which $M_{m}$ asymptotes. Nevertheless, the stratification of $M$ on outflow temperature is clearly seen from Figs. $13 \mathrm{~b}$ and $13 \mathrm{~d}$. Generally speaking, the Lilly model is a plausible approach for the steady-state TC structures and intensities given its simple governing equations, which are easier to understand mathematically and physically.

\section{d. The validity of (2) in the CM1 results}

As discussed in section 2a, the original L79 and L86 use the unperturbed environmental sounding for $T_{\infty}\left(s^{*}\right)$. However, (2) indicates the internal connection between $T_{\infty}\left(s^{*}\right)$ and the storm circulation, which means $T_{\infty}\left(s^{*}\right)$ should be determined by the storm itself, similar in concept to ER11, although according to (2), it is the boundary layer that sets $T_{\infty}\left(s^{*}\right)$, rather than the mixing in the TC outflow region as proposed in ER11. Following the discussion in section $2 \mathrm{a}$, for the flow rising out of the boundary layer (which is assumed to have $s^{*}=s$ above the boundary layer), (2) implies an internal consistency between $T_{\infty}$ and the distributions of $s^{*}$ and $E+(1 / 2) f M$ "upstream" such that $T_{\infty}\left(s^{*}\right)=d[E+(1 / 2) f M] / d s^{*}$. To evaluate the validity 

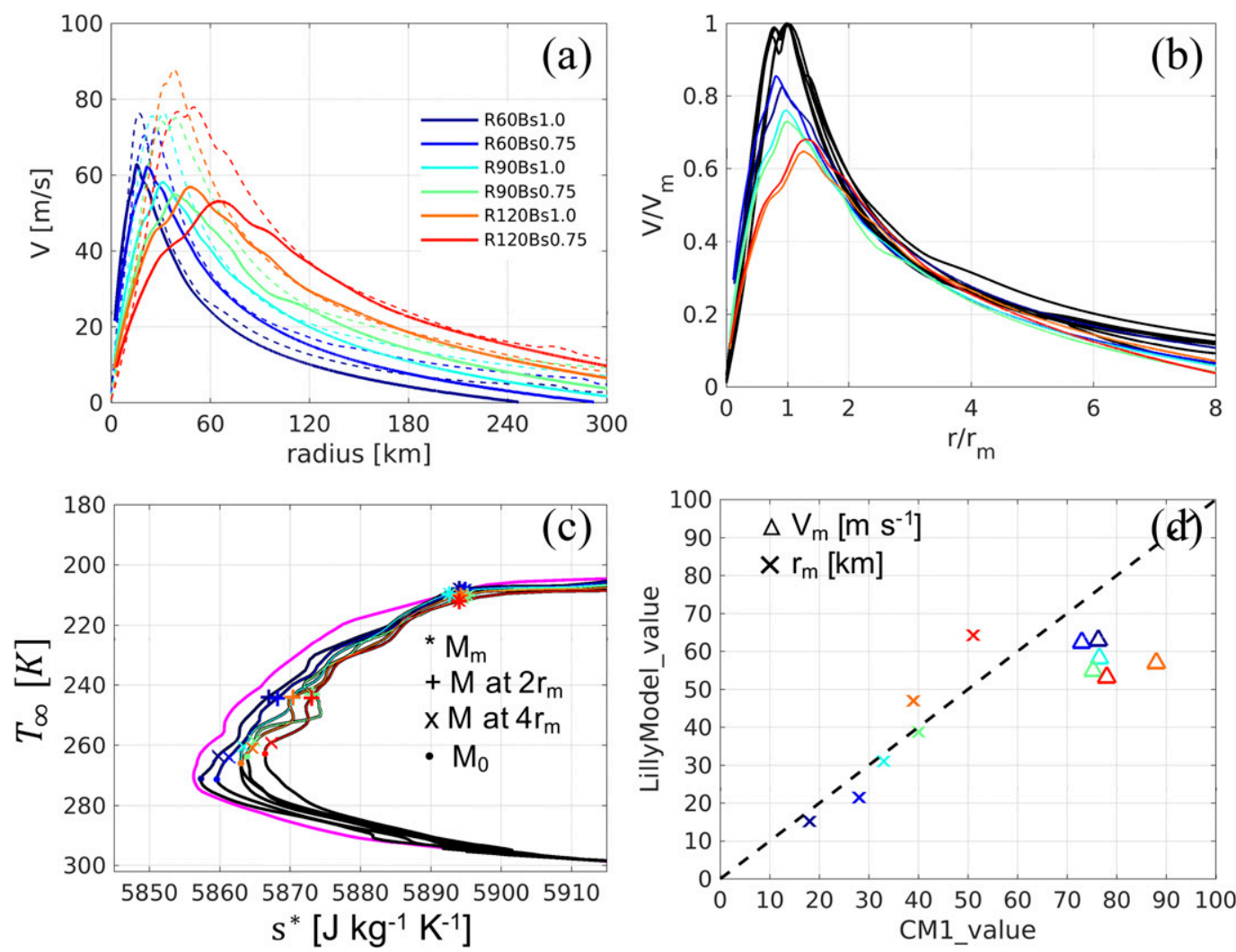

FIG. 12. Shown are the (a) radial profiles of tangential winds at the height of minimum $s^{*}$ in the sounding $T_{\infty}\left(s^{*}\right)$ with (dashed lines for the CM1 results and solid lines for the Lilly model results); (b) normalized version of (a) using CM1 $V_{m}$ and $r_{m}$ (black lines for the CM1 results, colored lines for the Lilly model results); (c) input function $T_{\infty}\left(s^{*}\right)$ used in the Lilly model (colored lines): black lines are the soundings at $r_{0}$ in the CM1 simulations, the Jordan sounding is in magenta color, the star denotes where the $M_{m}$ surface intersects with the sounding, the plus sign indicates the intersection of the $M$ surface across $2 r_{m}$ and the sounding, the cross shows the intersection of the $M$ surface across $4 r_{m}$ and the sounding, and the dot is where the $M_{0}$ surface intersects with the sounding; and (d) Lilly model $V_{m}$ and $r_{m}$ plotted against CM1 $V_{m}$ and $r_{m}$.

of (2), the $E+(1 / 2) f M$ and actual $s$ surfaces from the two CM1 simulations are shown in Figs. 14a and $14 \mathrm{~b}$ as well as the trajectory that passes through $r_{m}$; it is clear that the $E+(1 / 2) f M$ and $s$ surfaces are congruent over some distance surrounding the specified trajectory on which $s^{*} \cong s$. This congruency ensures that $d[E+(1 / 2) f M] / d s^{*}$ is at least approximately constant along an $s^{*}$ surface. To further investigate the validity of (2), Figs. $14 \mathrm{c}$ and $14 \mathrm{~d}$ show $E+(1 / 2) f M$ as a function of $s^{*}$ along two cross sections: one is a vertical section at some radius inside $r_{0}$ (i.e., in the outflow region, "downstream"), while the other is a horizontal section in the eyewall-updraft region (i.e., near the boundary layer top "upstream," defined by the minimum $s^{*}$ level in the temperature profile at $r_{0}$ as shown in Fig. 12c). In both small- and large-storm cases, the dependence of $E+(1 / 2) f M$ on $s^{*}$ is qualitatively the same at these two different sections, although there is a net reduction of $E+(1 / 2) f M$ on the same $s^{*}$ surfaces going from the upstream to the downstream section (which may indicate small-scale mixing and energy loss between these locations, possibly consistent with ER11). The direct calculation of $d[E+(1 / 2) f M] / d s^{*}$ has large artificial oscillations due to the uneven distribution and small number of data points in the model grids for the air rising out of the boundary layer. To avoid the unwanted fluctuations and obtain a smoother $d[E+(1 / 2) f M] / d s^{*}$, a sixth-degree polynomial is fit to the data points shown in Figs. $14 \mathrm{c}$ and $14 \mathrm{~d}$. The evenly distributed $[E+(1 / 2) f M]\left(s^{*}\right)$ is obtained for a $1 \mathrm{~J} \mathrm{~kg}^{-1} \mathrm{~K}^{-1}$ interval in $s^{*}$ using the fitted polynomial; $d[E+$ $(1 / 2) f M] / d s^{*}$ is calculated from a seven-point linear regression of the fitted data. Figures $14 \mathrm{e}$ and $14 \mathrm{f}$ show that the upstream $d[E+(1 / 2) f M] / d s^{*}$ in the outflow region (vertical yellow lines in Figs. 14a,b) agrees quantitatively well with the temperature sounding at $r_{0}$ and that both are significantly different from the unperturbed environmental sounding (the Jordan sounding). We note that $T_{\infty}\left(s^{*}\right)$ calculated from the upstream $d[E+(1 / 2) f M] / d s^{*}$ at the boundary layer top (horizontal yellow lines in Figs. 14a,b) exhibits a larger discrepancy with the temperature sounding at $r_{0}$. We also note that this discrepancy is somewhat larger for R60Bs 1.0 compared with R120Bs 0.75 , which could be the result of the low resolution in the eyewall region given the very small storm in the R60Bs1.0 case (only four data points in the eyewall-updraft region). Nevertheless, the dependence of $d[E+(1 / 2) f M] / d s^{*}$ on $s^{*}$ is captured qualitatively in the 

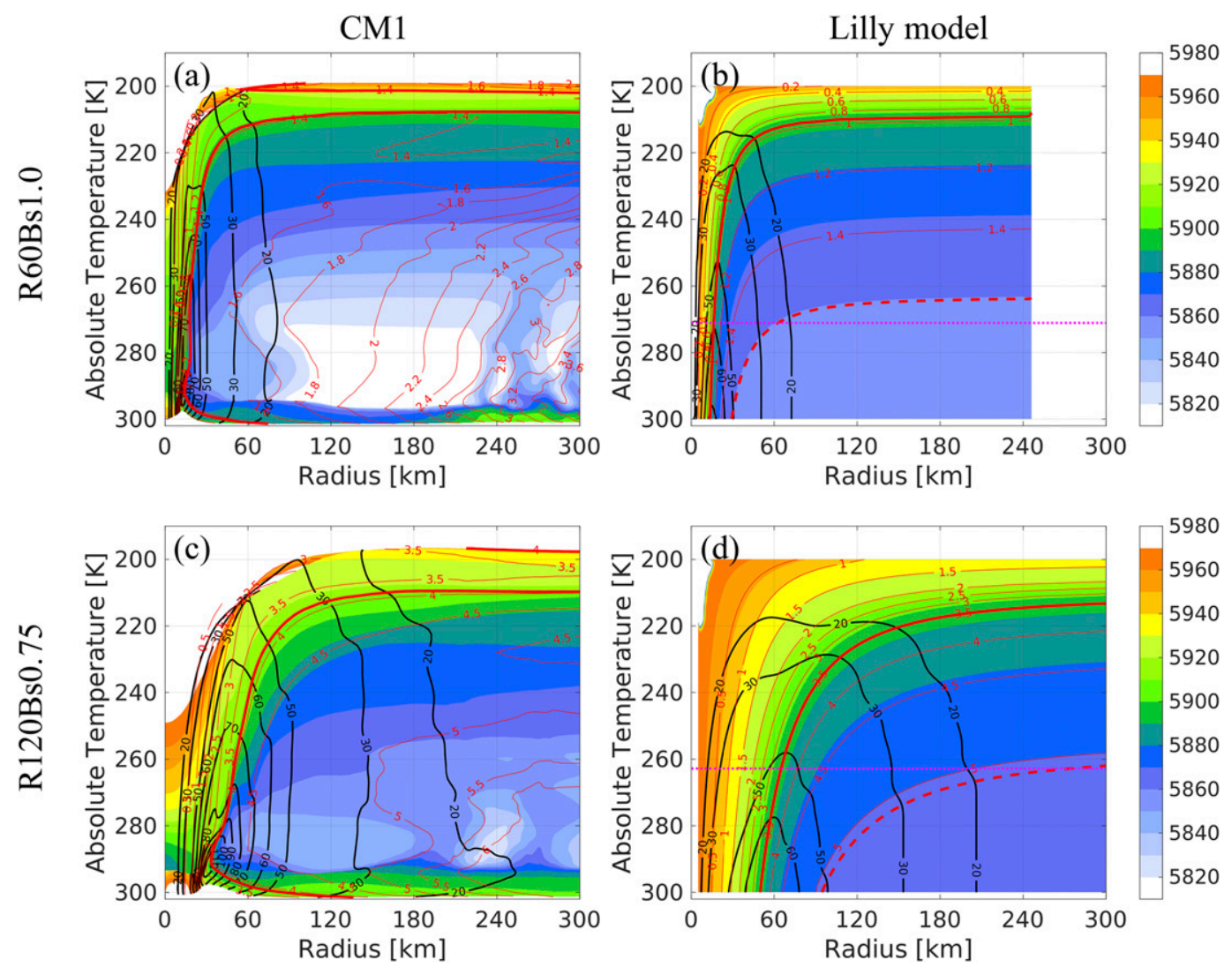

FIG. 13. Entropy surfaces (shading, actual entropy for CM1 results and saturation entropy for the Lilly model results), tangential winds (black contours), $M$ surfaces (thin red contours, unit: $10^{6} \mathrm{~m}^{2} \mathrm{~s}^{-1}$ ), and $M_{m}$ (thick red contour) in temperature-radius coordinate. (a),(b) R60Bs1.0; (c),(d) R120Bs0.75. (left) The results from CM1; (right) the results from the Lilly model. The red dashed lines in the right column denote the $M$ surfaces that pass across $4 r_{m}$ at the boundary layer top. The horizontal dotted magenta lines in the right column indicate the minimum $s^{*}$ levels shown in Fig. 12c.

upstream locations in both cases. Figure 14 adds weight to the idea that TCs are modifying their downstream outflow in such a way that $T_{\infty}\left(s^{*}\right)$ is close to $d[E+(1 / 2) f M] / d s^{*}$.

Finally, we note that the effect of the environment on the outflow temperature profile is through the height of the tropopause. The physical constraint is that the air cannot rise much above the tropopause level. Denote by $s_{m}^{*}$ the $s^{*}$ surface that starts at location of $V_{m}\left(r_{m}, p_{m}\right)$ and asymptotically reaches a height $p_{\infty}\left(s_{m}^{*}\right)$ for $r \gg r_{m}$ in the outflow region. Conservation of $s_{m}^{*}$ implies

$c_{p} \ln T_{m}+\frac{L_{o} q_{m}}{T_{m}}-R \ln p_{m}=c_{p} \ln T_{\infty}\left(s_{m}^{*}\right)+\frac{L_{o} q_{\infty}\left(s_{m}^{*}\right)}{T_{\infty}\left(s_{m}^{*}\right)}-R \ln p_{\infty}\left(s_{m}^{*}\right)$,

where $\left(T_{m}, p_{m}, q_{m}\right)$ and $\left(T_{\infty}, p_{\infty}, q_{\infty}\right)$ are the temperature, pressure, and mixing ratio at $\left(r_{m}, p_{m}\right)$ and in the outflow region, respectively. The tropopause constraint makes $p_{\infty}\left(s_{m}^{*}\right)$ approximately equal to the environmental tropopause pressure $(\sim 153 \mathrm{hPa}$ in the CM1 simulations) so that $T_{\infty}\left(s_{m}^{*}\right)$ can be obtained from (50) given $\left(T_{m}, p_{m}, q_{m}\right)$. For example, from simulation R120Bs0.75, $T_{m} \approx 288 \mathrm{~K}, p_{m} \approx 774 \mathrm{hPa}$, and $q_{m} \approx 14.3 \mathrm{~g} \mathrm{~kg}^{-1}$ at the boundary layer top $(z=1.55 \mathrm{~km})$ with $p_{\infty}\left(s_{m}^{*}\right) \approx 153 \mathrm{hPa}$ and $q_{\infty}\left(s_{m}^{*}\right)$ neglected in the outflow region, $(50)$ gives $T_{\infty}\left(s_{m}^{*}\right) \approx 206 \mathrm{~K}$, which is close to the $T_{\infty}\left(s_{m}^{*}\right)(210 \mathrm{~K})$ in the simulation. In general, $T_{m}, p_{m}$, and $q_{m}$ are not known without a complete theoretical treatment of the boundary layer, but the information must be contained in (2) since

$$
T_{\infty}\left(s_{m}^{*}\right)=\left.\frac{d\left(E+\frac{1}{2} f M\right)}{d s^{*}}\right|_{s^{*}=s_{m}^{*}} .
$$

Further research on the determinants of the relation among $s^{*}, M$, and $E$ through a boundary layer model and the relative contributions to $T_{\infty}\left(s^{*}\right)$ from outflow turbulent mixing is suggested.

\section{Summary}

This work revisits the steady-state axisymmetric tropical cyclone theory developed by D. K. Lilly. The Lilly model utilizes the gradient wind and hydrostatic balance with $M$ and $s^{*}$ conserved along streamlines to constrain the storm's dynamic and thermodynamic structures between the boundary layer top and the upper-level outflow downstream at large radii. 

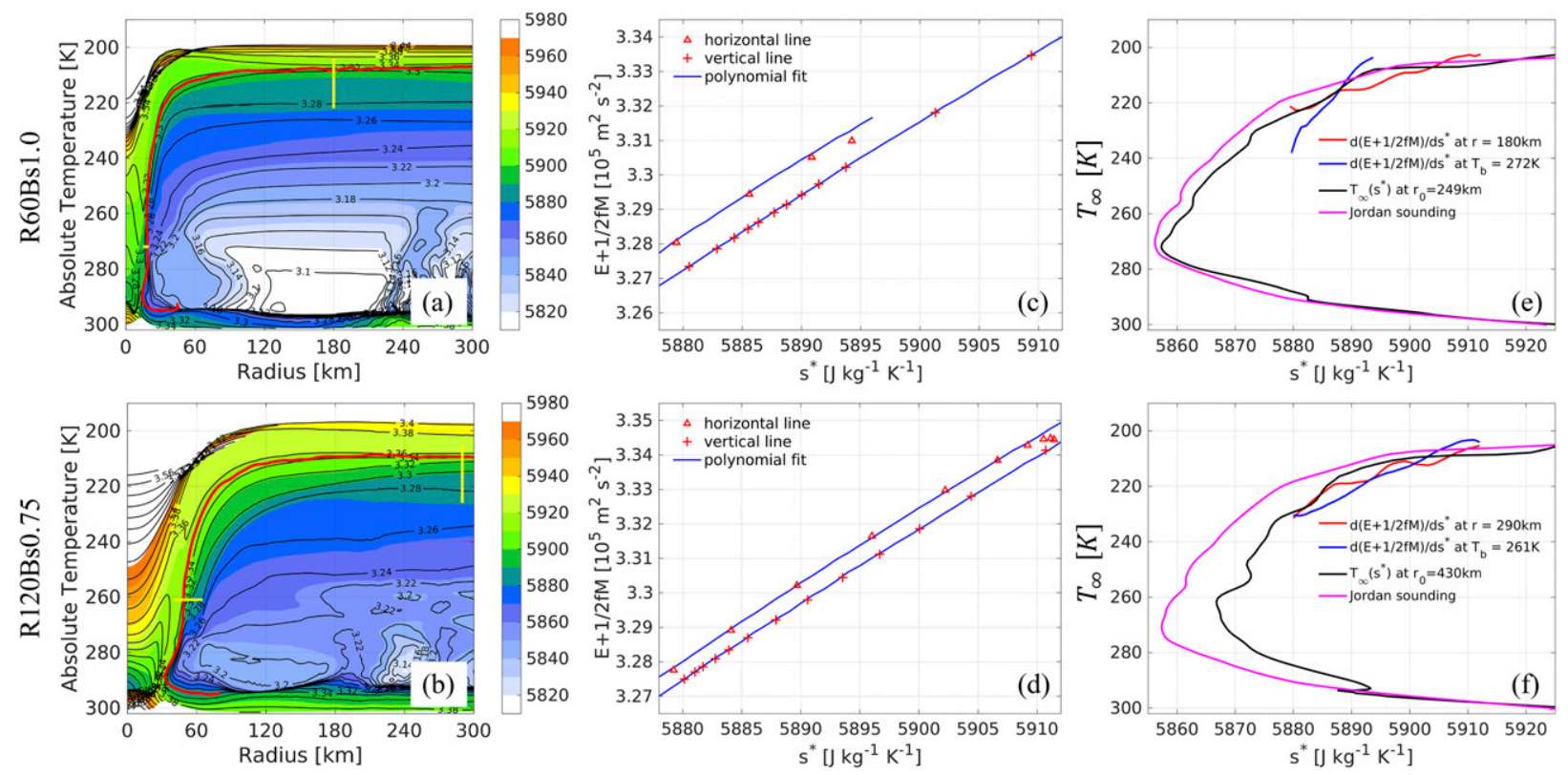

FIG. 14. (a),(b) Vertical cross sections of actual entropy $s$ (shading), $E+(1 / 2) f M$ (black contours, unit: $10^{5} \mathrm{~m}^{2} \mathrm{~s}^{-2}$ ), and the trajectory that passes through the radius of maximum wind at $1.55 \mathrm{~km}$ (thick red line); the thick yellow lines indicate the cross sections where the data in (c) and (d) are from. (c),(d) $E+(1 / 2) f M$ as a function of $s^{*}$ from the horizontal (triangle) and vertical (plus) yellow lines in (a) and (b); the blue lines indicate the sixth-degree polynomial fit. (e),(f) Vertical temperature profiles at $r_{0}$ (black lines), $d[E+(1 / 2) f M] / d s^{*}$ calculated from fitted polynomial in (c) and (d) (red lines for the vertical profile, blue lines for the horizontal profile); the unperturbed environmental (Jordan) sounding is shown in magenta. Rows show results for (a),(c),(e) R60Bs1.0 and (b),(d),(f) R120Bs0.75. All calculations are from the CM1 simulations.

For further understanding of this model, following Lilly, we reproduce and somewhat generalize his original analytic solutions for simplified cases. The dependence of the steady-state maximum intensity on the thermodynamic sounding is clearly seen even in the simplest case of a linear function, (19), with constant sea surface saturation entropy and no Coriolis term. Sensitivity tests using an exponential sounding and the full Lilly model show that reasonable variations of the parameters, $s_{s 0}^{*}, s_{0}^{*}, T_{t}$, and $T_{b}$, can only modify the tangential wind profile near the radius of maximum wind, while the change of initial input $M_{0}$ can alter the tangential wind profile at all radii in physical space but not near the radius of maximum wind in the normalized space. The most interesting finding is that the normalized tangential wind profiles can only be changed by the shape of the thermodynamic sounding (the $B$ parameter in exponential soundings) and supports the discussion of this point in ER11 (p. 2237). Adjusting other parameters only produces negligible differences in the normalized tangential wind profiles.

To test the versatility of the Lilly model in representing more realistic steady-state TCs, we also performed a comparison between the steady-state tangential wind radial structures from the Lilly model and those from the axisymmetric CM1 simulations. Generally speaking, the results indicate that the Lilly model can capture most of the inner-core characteristic features of different steady-state TCs not only at the boundary layer top but also vertically extending to the top of the troposphere.

Finally, the fundamental assumption of the Lilly model is that the outflow profile $T_{\infty}\left(s^{*}\right)$ is given, which greatly simplifies the mathematical problem. The original L79/L86 assume $T_{\infty}\left(s^{*}\right)$ is set by the unperturbed environment. On further consideration of the governing Eq. (1), it is deduced here that $T_{\infty}\left(s^{*}\right)$ is determined internally through the relation (2), the validity of which has been fortified by the evidence from the CM1 simulations in section $5 \mathrm{~d}$. At the same time, the environmental constraint on $T_{\infty}\left(s^{*}\right)$ comes from the tropopause height which, through the conservation of $s^{*}$, determines $T_{\infty}\left(s_{m}^{*}\right)$. This $T_{\infty}\left(s_{m}^{*}\right)$ in turn is identical to (2) for a steady-state solution, as discussed in section $5 \mathrm{~d}$. We believe the present exposition of the Lilly model provides a clearer understanding of the role of the outflow temperature profile $T_{\infty}\left(s^{*}\right)$ in axisymmetric, steady-state TC models.

Given the highly idealized assumptions, there are some further caveats to keep in mind with respect to the Lilly model. One limitation is that the sounding of $T_{\infty}\left(s^{*}\right)$ needs to be monotonically decreasing with increasing $s^{*}$, which leads to an unrealistic boundary layer depth (indicated by the dot magenta lines in Figs. 13b,d). Another limitation (shared by ER11) is that the assumptions of gradient wind and hydrostatic balance preclude supergradient winds. These deficiencies notwithstanding, we believe the Lilly model is a useful approach to access the essence of steadystate TC structures. The discrepancies between the Lilly model and CM1 results as well as the potential connection between the $T_{\infty}\left(s^{*}\right)$ in the TC outflow region and the $T_{\infty}\left(s^{*}\right)$ in the unperturbed environment will be investigated in a follow-up study. 
Acknowledgments. Authors Dr. Tao and Dr. Bell are supported by Office of Naval Research Awards N000141613033 and N000142012069, and National Science Foundation Award AGS-1701225. The contribution of Dr. R. Rotunno to this work is supported by the National Center for Atmospheric Research (NCAR), which is a major facility sponsored by the National Science Foundation under Cooperative Agreement 1852977. The authors also thank Dr. Christopher A. Davis (NCAR), reviewer Dr. Kerry A. Emanuel (MIT), and two other anonymous reviewers for their insightful comments, which greatly improved this manuscript. Computing was performed on a local computer server at the Department of Atmospheric Science, Colorado State University. The test code for the Lilly model is available upon request.

\section{REFERENCES}

Batchelor, G. K., 1967: An Introduction to Fluid Dynamics. Cambridge University Press, 615 pp.

Bengtsson, L., K. I. Hodges, M. Esch, N. Keenlyside, L. Kornbleuh, J. J. Luo, and T. Yamagata, 2007: How may tropical cyclones change in a warmer climate? Tellus, 59A, 539-561, https:// doi.org/10.1111/j.1600-0870.2007.00251.x.

Bryan, G. H., 2008: On the computation of pseudoadiabatic entropy and equivalent potential temperature. Mon. Wea. Rev., 136, 5239-5245, https://doi.org/10.1175/2008MWR2593.1.

— the maximum intensity of tropical cyclones. J. Atmos. Sci., 66, 3042-3060, https://doi.org/10.1175/2009JAS3038.1.

— in axisymmetric numerical model simulations. Mon. Wea. Rev., 137, 1770-1789, https://doi.org/10.1175/2008MWR2709.1.

Chan, K. T. F., and J. C. L. Chan, 2014: Impacts of initial vortex size and planetary vorticity on tropical cyclone size. Quart. J. Roy. Meteor. Soc., 140, 2235-2248, https://doi.org/10.1002/qj.2292.

Chavas, D. R., N. Lin, and K. Emanuel, 2015: A model for the complete radial structure of the tropical cyclone wind field. Part I: Comparison with observed structure. J. Atmos. Sci., 72, 3647-3662, https://doi.org/10.1175/JAS-D-15-0014.1.

Emanuel, K. A., 1986: An air-sea interaction theory for tropical cyclones. Part I: Steady-state maintenance. J. Atmos. Sci., 43, 585-605, https://doi.org/10.1175/1520-0469(1986)043<0585: AASITF $>2.0 . \mathrm{CO} ; 2$.

, 2004: Tropical cyclone energetics and structure. Atmospheric Turbulence and Mesoscale Meteorology, E. Fedorovich, R. Rotunno, and B. Stevens, Eds., Cambridge University Press, 165-192.

- 2005: Increasing destructiveness of tropical cyclones over the past 30 years. Nature, 436, 686-688, https://doi.org/ 10.1038/nature03906.

_ , and R. Rotunno, 2011: Self-stratification of tropical cyclone outflow. Part I: Implications for storm structure. J. Atmos. Sci., 68, 2236-2249, https://doi.org/10.1175/JAS-D-10-05024.1.

$\longrightarrow,-$, and D. K. Lilly, 1985: An air-sea interaction theory for tropical cyclones. Preprints, 16th Conf. on Hurricanes and Tropical Meteorology, Houston, TX, Amer. Meteor. Soc., 27-28.

Holland, G. J., 1997: The maximum potential intensity of tropical cyclones. J. Atmos. Sci., 54, 2519-2541, https://doi.org/10.1175/ 1520-0469(1997)054<2519:TMPIOT>2.0.CO;2.

Iman, R. L., M. E. Johnson, and C. C. Watson, 2005: Sensitivity analysis for computer model projections of hurricane losses. Risk Anal., 25, 1277-1297, https://doi.org/10.1111/j.15396924.2005.00673.x.

Irish, J. L., D. T. Resio, and J. J. Ratcliff, 2008: The influence of storm size on hurricane surge. J. Phys. Oceanogr., 38, 20032013, https://doi.org/10.1175/2008JPO3727.1.

Lilly, D. K., and K. Emanuel, 1985: A steady-state hurricane model. Preprints, 16th Conf. on Hurricanes and Tropical Meteorology, Houston, TX, Amer. Meteor. Soc., 142-143.

Long, R. R., 1955: Some aspects of the flow of stratified fluids. III: Continuous density gradients. Tellus, 7, 341-357, https://doi.org/ 10.3402/tellusa.v7i3.8900.

Peng, K., R. Rotunno, G. H. Bryan, and J. Fang, 2019: Evolution of an axisymmetric tropical cyclone before reaching slantwise moist neutrality. J. Atmos. Sci., 76, 1865-1884, https://doi.org/ 10.1175/JAS-D-18-0264.1.

Shutts, G. J., 1981: Hurricane structure and the zero potential vorticity approximation. Mon. Wea. Rev., 109, 324-329, https://doi.org/10.1175/1520-0493(1981)109<0324:HSATZP > 2.0.CO;2.

Stern, D. P., and D. S. Nolan, 2009: Reexamining the vertical structure of tangential winds in tropical cyclones: Observations and theory. J. Atmos. Sci., 66, 3579-3600, https://doi.org/10.1175/ 2009JAS2916.1.

Tao, D., K. Emanuel, F. Zhang, R. Rotunno, M. M. Bell, and R. G. Nystrom, 2019: Evaluation of the assumptions in the steady-state tropical cyclone self-stratified outflow using three-dimensional convection-allowing simulations. J. Atmos. Sci., 76, 2995-3009, https://doi.org/10.1175/JAS-D-19-0033.1.

_ - M. Bell, R. Rotunno, and P. J. Van Leeuwen, 2020: Why do the maximum intensities in modeled tropical cyclones vary under the same environmental conditions? Geophys. Res. Lett., 47, e2019GL085980, https://doi.org/10.1029/2019g1085980.

$\mathrm{Xu}$, J., and Y. Wang, 2010: Sensitivity of the simulated tropical cyclone inner-core size to the initial vortex size. Mon. Wea. Rev., 138, 4135-4157, https://doi.org/10.1175/2010MWR3335.1.

— tropical cyclone intensification rate on the storm intensity and size in the North Atlantic. Wea. Forecasting, 30, 692-701, https://doi.org/10.1175/WAF-D-14-00141.1.

—_, and — , 2018: Effect of the initial vortex structure on intensification of a numerically simulated tropical cyclone. J. Meteor. Soc. Japan, 96, 111-126, https://doi.org/10.2151/ jmsj.2018-014.

Yih, C. S., 1965: Dynamics of Nonhomogeneous Fluids. MacMillan, $306 \mathrm{pp}$.

Ying, Y., and Q. Zhang, 2012: A modeling study on tropical cyclone structural changes in response to ambient moisture variations. J. Meteor. Soc. Japan, 90, 755-770, https://doi.org/10.2151/ jmsj.2012-512. 